\title{
Enhanced Sensitivity of Nonsmall Cell Lung Cancer with Acquired Resistance to Epidermal Growth Factor Receptor-Tyrosine Kinase Inhibitors to Phenformin: The Roles of a Metabolic Shift to Oxidative Phosphorylation and Redox Balance
}

\author{
Suntae Kim, ${ }^{1}$ Ji Hye Im, ${ }^{1,2}$ Wan Kyu Kim $\mathbb{D}^{3}{ }^{3}$ Young Jae Choi, ${ }^{4,5}$ Ji-Yoon Lee, \\ Sang Kyum Kim, ${ }^{4}$ Sun Jo Kim, ${ }^{1}$ Sung Won Kwon, ${ }^{1}$ and Keon Wook Kang ${ }^{1}{ }^{1}$ \\ ${ }^{1}$ College of Pharmacy and Research Institute of Pharmaceutical Sciences, Seoul National University, Seoul 08826, Republic of Korea \\ ${ }^{2}$ Department of Cancer Control, National Cancer Center Graduate School of Cancer Science and Policy, National Cancer Center, \\ Goyang 10408, Republic of Korea \\ ${ }^{3}$ Department of Life Sciences, Ewha Womans University, Seoul 03760, Republic of Korea \\ ${ }^{4}$ College of Pharmacy, Chungnam National University, Daejeon 34134, Republic of Korea \\ ${ }^{5}$ Bioanalysis and Pharmacokinetics Research Group, Korea Institute of Toxicology, Daejeon 34114, Republic of Korea
}

Correspondence should be addressed to Keon Wook Kang; kwkang@snu.ac.kr

Received 28 May 2021; Accepted 29 June 2021; Published 29 July 2021

Academic Editor: Jolanta Czuczejko

Copyright (c) 2021 Suntae Kim et al. This is an open access article distributed under the Creative Commons Attribution License, which permits unrestricted use, distribution, and reproduction in any medium, provided the original work is properly cited.

Background. Although the efficacy of epidermal growth factor receptor-tyrosine kinase inhibitor (EGFR- TKI) therapy has been proven in non-small cell lung cancer (NSCLC) patients, acquired resistance to EGFR-TKIs presents a serious clinical problem. Hence, the identification of new therapeutic strategy is needed to treat EGFR-TKI-resistant NSCLC. Methods. Acquired EGFRTKI-resistant lung cancer cell lines (HCC827, H1993, and H292 cells with acquired resistance to gefitinib or erlotinib) were used for cell-based studies. IncuCyte live cell analysis system and XFp analyzer were used for the determination of cell proliferation and energy metabolism, respectively. In vivo anticancer effect of phenformin was assessed in xenografts implanting HCC827 and gefitinib-resistant HCC827 (HCC827 GR) cells. Results. HCC827 GR and erlotinib-resistant H1993 (H1993 ER) cells exhibited different metabolic properties compared with their respective parental cells, HCC827, and H1993. In EGFR-TKI-resistant NSCLC cells, glycolysis markers including the glucose consumption rate, intracellular lactate level, and extracellular acidification rate were decreased; however, mitochondrial oxidative phosphorylation (OXPHOS) markers including mitochondria-driven ATP production, mitochondrial membrane potential, and maximal OXPHOS capacity were increased. Cell proliferation and tumor growth were strongly inhibited by biguanide phenformin via targeting of mitochondrial OXPHOS complex 1 in EGFR-TKI-resistant NSCLC cells. Inhibition of OXPHOS resulted in a reduced $\mathrm{NAD}^{+} / \mathrm{NADH}$ ratio and intracellular aspartate levels. Recovery of glycolysis by hexokinase 2 overexpression in erlotinibresistant H292 (H292 ER) cells significantly reduced the anticancer effects of phenformin. Conclusion. Long-term treatment with EGFR-TKIs causes reactivation of mitochondrial metabolism, resulting in vulnerability to OXPHOS inhibitor such as phenformin. We propose a new therapeutic option for NSCLC with acquired EGFR-TKI resistance that focuses on cancer metabolism.

\section{Background}

Epidermal growth factor receptor-tyrosine kinase inhibitors (EGFR-TKIs) such as gefitinib and erlotinib have generally been used in non-small cell lung cancer (NSCLC) patients as first-line targeted therapy since 2003, and their prognosis has significantly improved with the targeted therapy. Unfortunately, long-term treatment with EGFR-TKIs frequently 
causes resistance to targeted therapy, which led to the development of second- and third-generation EGFR-TKIs that avoid point mutations in the EGFR tyrosine kinase domain [1]. The T790M point mutation in EGFR exon 20 was reported as a major cause of EGFR-TKI resistance [1, 2]. Consequently, diverse resistance research was conducted on the H1975 NSCLC cell line carrying the T790M mutation, and a third-generation EGFR-TKI, osimertinib (Tagrisso ${ }^{\circledR}$ ), which targets T790M, has been approved by the United States Food and Drug Administration as first-line therapy for metastatic lung cancer patients with an EGFR exon 19 deletion or L858R mutation [3]. However, acquired resistance to EGFR-TKIs may not be solely due to point mutations in some patients [2]. Although several molecular mechanisms for acquired resistance to EGFR-TKIs have been suggested, clinically available new therapeutic strategies are still needed [1].

Because a principal hallmark of cancer cells is rapid growth, a sufficient supply of nutrients is a critical requirement for cancer cells. Therefore, understanding cell typespecific metabolic processes that consume or use nutrients in cancer is important and could offer potential target(s) for cancer chemotherapy. After the discovery of the Warburg effect (metabolic dependency on glycolysis even under aerobic conditions), metabolic reprogramming in tumors has been extensively studied, and several clinically effective targets for controlling cancer cell-specific metabolism have been identified $[4,5]$. In the present study, a new therapeutic strategy for treating EGFR-TKI-resistant NSCLC that focuses on cancer metabolism is proposed and the pharmacological efficacy of phenformin, a biguanide agent was elucidated.

\section{Materials and Methods}

2.1. Reagents and Antibodies. Antibodies recognizing Hexokinase I (C35C4), Hexokinase II (C64G5), phosphopyruvate kinase isozymes M2 (PKM2) (Tyr105), PKM2 (D78A4), $\alpha$-tubulin, phospho-EGFR (Tyr1068) (1H12), phospho-AKT (Ser473), and phospho-p44/42 mitogenactivated protein kinase (MAPK) (Thr202/Tyr204) (20G11) were purchased from Cell signaling Technology (Danvers, MA, USA). Anti-Pyruvate Dehydrogenase E1-alpha subunit (S293) and total OXPHOS human antibody cocktail were obtained from Abcam (Cambridge, United Kingdom). Antiglyceraldehyde-3-phosphate dehydrogenase (GAPDH) was supplied from Millipore (Burlington, MA, USA) and anti-Flag was purchased from Sigma Aldrich (St. Louis, MO, USA). Epidermal growth factor (EGF), sodium 2oxobutyrate $(\mathrm{AKB})$, and L-aspartic acid were supplied from Sigma Aldrich. Gefitinib and osimertinib were obtained from MedChemExpress (Monmouth Junction, NJ, USA). Erlotinib, phenformin, and rotenone were purchased from Cayman (Ann Arbor, MI, USA).

2.2. Cell Culture and Establishment of Stably Hexokinase II Overexpressing H292 ER Cells. Human lung cancer cells H1993, H1993 ER, H292, and H292 ER cells were kind gifts of Dr. Sang Kook Lee (Seoul National University, Seoul, Republic of Korea). HCC827, HCC827 GR, H1975, H1993,
H1993 ER, H292, and H292 ER cells were cultured in RPMI 1640 medium (Hyclone, Logan, UT, USA) with 10\% fetal bovine serum (Biowest, MO, USA) and 1\% penicillin/streptomycin $(100 \mathrm{U} / \mathrm{mL}$, Hyclone). All cells were maintained in an incubator humidified $5 \% \mathrm{CO}_{2}$ at $37^{\circ} \mathrm{C}$.

To establish hexokinase II stably overexpressing cells, pCAG-Flag-HK2-IRES-Blas plasmid was transfected to H292 ER cells using Lipofectamine 2000 as specified by the manufacturer's instruction (Invitrogen, Carlsbad, CA, USA). Colonies were selected by incubation with blasticidin (15 $\mu \mathrm{g} / \mathrm{mL}$, Invitrogen). pCAG-Flag-IRES-Blas vector was used for mock transfection. pCAG-Flag-IRES-Blas and pCAG-Flag-HK2-IRES-Blas plasmids were kindly donated from Dr. Hong-Duk Youn (Department of Molecular Medicine \& Biopharmaceutical Sciences, Graduate School of Convergence Science, Seoul National University, Seoul, Republic of Korea).

2.3. Cell Proliferation. Cells were seeded in a 96-well plate $\left(2 \times 10^{3}\right.$ cells/well $)$ and cultured for $72 \mathrm{~h}$. To determine cell proliferation, real-time scanned phase-contrast images were acquired and integrated confluence was analyzed by IncuCyte $^{\circledR}$ ZOOM $^{\mathrm{TM}}$ Live Cell Analysis system (Essen BioScience, Ann Arbor, MI, USA).

2.4. Genomic DNA Sequencing. Genomic DNA from lung cancer cell lines was extracted with lysis buffer (10\% sodium dodecyl sulfate (SDS), $100 \mathrm{mM} \mathrm{NaCl}, 100 \mathrm{mM}$ ethylenediaminetetraacetic acid (EDTA), $50 \mathrm{mM}$ Tris ( $\mathrm{pH} 8.0)$ ) and proteinase $\mathrm{K}(15 \mathrm{mg} / \mathrm{mL})$. Exon 18-21 region of EGFR gene was amplified by polymerase-chain-reaction (PCR). Thermal cycler settings include an initial denaturation at $95^{\circ} \mathrm{C}$ for $3 \mathrm{~min}$ followed by 40 cycles of denaturation at $95^{\circ} \mathrm{C}$ for $20 \mathrm{sec}$, annealing at $59.1^{\circ} \mathrm{C}$ for $10 \mathrm{sec}$, and extension at $72^{\circ} \mathrm{C}$ for $30 \mathrm{sec}$. Primers for exon 18 (sense: CAAATGAGCTG GCAAGTGCCGTGTC, antisense: GAGTTTCCCAAACA CTCAGTGAAAC), exon 19 (sense: GCAATATCAGCCTT AGGTGCGGCTC, antisense: CATAGAAAGTGAACAT TTAGGATGTG), exon 20 (sense: CCATGAGTACGTAT TTTGAAACTC, antisense: CATATCCCCATGGCAAACT CTTGC), and exon 21 (sense: CTAACGTTCGCCAGCC ATAAGTCC, antisense: GCTGCGAGCTCACCCAGAATG TCTGG) were used, and capillary electrophoresis sequencing was performed in Macrogen (Seoul, South Korea).

2.5. Immunoblot Analysis. After washing with cold sterile PBS, cells were lysed with lysis buffer containing $20 \mathrm{mM}$ Tris- $\mathrm{Cl}$ ( $\mathrm{pH}$ 7.5), 1\% Triton $\mathrm{X}-100,137 \mathrm{mM}$ sodium chloride, $1 \mathrm{mM}$ sodium orthovanadate, $10 \%$ glycerol, $2 \mathrm{mM}$ EDTA, $25 \mathrm{mM} \beta$-glycerolphosphate, $1 \mathrm{mM}$ phenylmethylsulfonylfluoride, $2 \mathrm{mM}$ sodium inorganic pyrophosphate, and $1 \mu \mathrm{g} / \mathrm{mL}$ leupeptin. Total cell lysates were separated using SDS polyacrylamide gel and electrophoretically transferred to nitrocellulose membranes. After serial incubation of the membranes with primary antibodies and horseradish peroxidase- (HRP-) conjugated anti-IgG antibodies, the membranes were exposed to enhanced chemiluminescence (ECL) reagent (Millipore, Burlington, MA, USA), and images were obtained using LAS3000 mini (Fujifilm, Tokyo, Japan). 
2.6. 2-Deoxyglucose- (2-DG-) Uptake Assay. Cells seeded in a 96-well plate $\left(2 \times 10^{4}\right.$ cells/well $)$ were incubated with $1 \mathrm{mM}$ 2-DG for $10 \mathrm{~min}$. 2-deoxyglucose-6-phosphate (2-DGP) was determined using Glucose uptake-Glo assay kit (Promega, Madison, WI, USA) following the manufacturer's instruction.

2.7. Determination of $\mathrm{NAD}^{+} / \mathrm{NADH}$ Ratio. Cells were plated in a 96-well plate and treated with the indicated concentration of phenformin for $24 \mathrm{~h}$. Using $\mathrm{NAD}^{+} / \mathrm{NADH}$ Glo Assay (Promega), the $\mathrm{NAD}^{+} / \mathrm{NADH}$ ratio was calculated [6].

2.8. Determination of Glucose Consumption in Culture Medium. Cell culture media were collected $24 \mathrm{~h}$ after incubation, and a 1000 -fold diluted solution was achieved by adding ice-cold $50 \%$ methanol. The solution was introduced to 1 equivalent volume of chloroform for phase separation. Samples were vortexed for $10 \mathrm{~s}$ and subsequently centrifuged at $16,000 \mathrm{rcf}$ for $5 \mathrm{~min}$. The upper hydrophilic phase was transferred to a microcentrifuge tube, and the extraction was repeated once with the remaining lower phase. The collected hydrophilic phase was dried under gentle stream of nitrogen. Dried samples were reconstituted with $100 \mu \mathrm{L}$ methoxyamine hydrochloride $(20 \mathrm{mg} / \mathrm{mL}$, Sigma Aldrich) in pyridine and incubated at $37^{\circ} \mathrm{C}$ for $90 \mathrm{~min}$. Following cool-down, samples were introduced to $100 \mu \mathrm{L} \mathrm{N}, \mathrm{O}$-bis(trimethylsilyl)trifluoroacetamide solution with $1 \%$ trimethylchlorosilane (Sigma Aldrich) and derivatized at $60^{\circ} \mathrm{C}$ for $40 \mathrm{~min}$. Glucose contents were analyzed by the Shimadzu GCMS-QP2010 (Tokyo, Japan) system equipped with DB-5MS (30 m, $0.25 \mathrm{~mm}, 0.25 \mu \mathrm{m}$; Agilent Technologies, DE, USA). Inlet temperature was set at $270^{\circ} \mathrm{C}$, and the samples were injected in split mode $(1: 2)$. Column oven temperature was maintained as the following gradient: $0 \mathrm{~min}, 80^{\circ} \mathrm{C} ; 2 \mathrm{~min}, 80^{\circ} \mathrm{C}$; $7 \mathrm{~min}, 100^{\circ} \mathrm{C} ; 10 \mathrm{~min}, 100^{\circ} \mathrm{C} ; 35 \mathrm{~min}, 200^{\circ} \mathrm{C} ; 36 \mathrm{~min}, 200^{\circ} \mathrm{C}$; $48.5 \mathrm{~min}, 300^{\circ} \mathrm{C}$; and $50.5 \mathrm{~min}, 300^{\circ} \mathrm{C}$. Mass scan ranged from 40 to $600 \mathrm{~m} / \mathrm{z}$ with $3.06 \mathrm{scan} / \mathrm{s}$ of scan rate. Absolute quantitative values were calculated with peak area based on calibration curve by external glucose standard solutions and normalized by total protein contents.

2.9. Determination of Intracellular Lactate and Aspartate Levels. The sample injection volume was $5 \mu \mathrm{L}$, and peak separation was performed on a Hypersil GOLD $\mathrm{C}_{8}$ $(2.1 \times 150 \mathrm{~mm}, 5 \mu \mathrm{m}$, Thermo Scientific, Waltham, MA) maintained at $30^{\circ} \mathrm{C}$. The analysis was performed to gradient condition using Agilent 1290 infinity II system with autosampler, column oven, and binary pump (HPLC water containing $0.01 \%(v / v)$ formic acid, A; $100 \%$ methanol, B), and flow rate was $0.2 \mathrm{~mL} / \mathrm{min}$. LC-MS/MS data were acquired with an Applied Biosystems SCIEX 4000 QTRAP hybrid triple quadrupole-linear ion trap mass spectrometer equipped with a Turbo $\mathrm{V}$ ionization source. The detection was conducted using multiple reaction monitoring (MRM) of the transitions of $\mathrm{m} / \mathrm{z} 89>43$ for lactate, $\mathrm{m} / \mathrm{z} 132>88$ for aspartate, and $\mathrm{m} / \mathrm{z} 157>112$ for ${ }^{13} \mathrm{C}_{5}, \mathrm{D}_{5},{ }^{15} \mathrm{~N}$-glutamate (ISTD) in the negative ion mode. Acquisition and analysis data were performed with Analyst ${ }^{\circledR}$ software (ver.1.6.2; Applied Biosystems, Foster City, CA, USA).
2.10. Determination of Mitochondria ATP Production Rate, Oxygen Consumption Rate (OCR), and Extracellular Acidification Rate (ECAR). OCR and ECAR were determined using XFp analyzer (Seahorse Bioscience, North Billerica, MA, USA). XFp cell mito-stress test kit, XFp glycolysis stress test kit, and XFp Real-Time ATP rate assay kit (Seahorse Bioscience) were used to detect OCR, ECAR, and ATP ratio, respectively. All the reagents and assay conditions were followed by manufacturer's instructions.

2.11. RNA Preparation and RNA Sequencing. Total RNA was extracted from HCC827 and HCC827 GR cells using TRIzol reagent (Invitrogen). Purity and concentration of RNA samples were evaluated by NanoDrop Lite (Thermo Scientific, MA, USA), and transcriptome RNA-sequencing of the samples was performed by Macrogen.

2.12. Transmission Electron Microscopy. Cells were fixed with Karnovsky's fixative and fixed with $2 \%$ osmium tetroxide. $0.5 \%$ uranyl acetate was used for staining, and propylene oxide and ethanol were used for dehydration. By using Spurr's resin, cells were embedded and polymerized at $70^{\circ} \mathrm{C}$. After embedding, blocks were trimmed with ultramicrotome (EM UC7, Leica, Wetzler, Germany) and detected with Transmission Electron Microscope (JEM1010, JEOL, Tokyo, Japan).

2.13. Flow Cytometry Analysis to Determine Mitochondria Membrane Potential. Tetramethylrhodamine methyl ester perchlorate (TMRM) (Sigma-Aldrich, MO, USA) was prepared as $100 \mu \mathrm{M}$ stock solution in dimethyl sulphoxide. Cancer cells were incubated with $100 \mathrm{nM}$ TMRM for $30 \mathrm{~min}$ at $37^{\circ} \mathrm{C}$. After trypsin treatment, the detached stained cells were analyzed by Novocyte flow cytometer (Agilent, CA, USA).

2.14. Xenograft Assay. Five-week-old male Balb/c-nu mice were supplied from Raon Bio Inc. (Seoul, South Korea). Animal studies were performed according to the institute regulation and approval from Seoul National University Institutional Animal Care and Use Committee (Approval \#: SNU-170717-6-1). Mice were kept in SNU semipathogenfree animal facility, with five mice in each cage. After anesthesia with Zoletil ${ }^{\circledR} /$ Rompun $^{\circledR}$ mixture, $3.5 \times 10^{6}$ HCC827 or $3 \times 10^{6}$ HCC 827 GR cells were inoculated on right flanks of mice. One week after inoculation, the mice were randomly divided into two groups (vehicle control group and phenformin treatment group $(300 \mathrm{mg} / \mathrm{kg} /$ day, P.O.)). Animal number in xenograft study is 6-10 per group. Mice were monitored every other day, tumor length and width were detected by calipers, and tumor volume was calculated using the formula (length $\times$ width $\left.^{2}\right) \times 0.5$. Animals were sacrificed by carbon dioxide inhalation in euthanasia chamber.

2.15. Statistics. Student's $t$-test was performed to compare the difference between two groups. One-way ANOVA and Tukey's post hoc was used to analyze differences in multiple comparison. Statistical analysis was calculated using SigmaPlot (version 12.5). The statistical significance was accepted at $* P<0.05, * * P<0.01$, and $* * * P<0.001 ;{ }^{\#} P<0.05$, ${ }^{\# \#} P<0.01$, and ${ }^{\# \#} P<0.001$. 


\section{Results}

3.1. Long-Term Treatment with EGFR-TKIs Induces Acquired Resistance with Downregulation of EGFR Signaling in Lung Cancer Cells. Gefitinib-resistant NSCLC HCC827 (HCC827 GR) cells were established via long-term exposure of the cells to stepwise escalation of gefitinib. HCC827 GR cells exhibited resistance to gefitinib at a level similar to that in H1975 cells (T790M mutation). Other lung cancer cell lines, H1993 (NSCLC) and H292 (mucoepidermoid lung cancer) cells, also exhibited resistance to erlotinib after long-term treatment (H1993 ER and H292 ER, respectively) (Figure 1(a)). Osimertinib, a third-generation EGFR-TKI, strongly inhibited H1975 cell proliferation but marginally affected cell proliferation in the lung cancer cell lines with acquired EGFR-TKI resistance (HCC827 GR, H1993 ER, and H292 ER) (Figure 1(b)). Because various mutations in the EGFR tyrosine kinase domain associated with gefitinib resistance have been reported [7], DNA sequencing of EGFR exons 18 to 21 was performed for HCC827, HCC827 GR, H1975, H1993, H1993 ER, H292, and H292 ER cells. An exon 19 deletion mutation and inherited gefitinib resistance, T790M, were found in HCC827 and H1975 cells, respectively (Figure 1(c)). However, mutations were not detected in all the lung cancer cells with acquired EGFR-TKI resistance tested (Figure 1(c)). Furthermore, EGF-induced phosphorylation of EGFR (Tyr1068) and its downstream kinases, phosphorylation of AKT (Ser473) or p44/p42 MAPK (Thr202/Tyr204), was not observed or was weaker in the three acquired-resistance cells compared with their parental cell lines. In addition, the EGFR tyrosine kinase-inhibiting effects of gefitinib, erlotinib, and osimertinib were weaker (Figure 1(d)). Conversely, EGFR phosphorylation and its downstream signals were successfully suppressed by osimertinib but not by gefitinib or erlotinib in H1975 cells (T790M) (Figure 1(d)). The results showed that the acquisition of EGFR-TKI resistance by long-term treatment with gefitinib or erlotinib led to relatively minimal effects on EGFR activity, and these cells subsequently exhibited decreased sensitivity to EGFR-TKIs, even osimertinib.

3.2. Glycolysis Activity Is Decreased in Lung Cancer Cells with Acquired EGFR-TKI Resistance. EGFR signaling promotes aerobic glycolysis in triple-negative breast cancer (TNBC) [8], and the inhibition of EGFR reverses the Warburg effect and reactivates oxidative phosphorylation (OXPHOS) in NSCLC cells [9]. Because EGFR signaling is suppressed in the three acquired-resistance lung cancer cell lines (HCC827 GR, H1993 ER, and H292 ER), we examined glucose metabolism in these cancer cells. The 2-DG uptake assay showed that glucose uptake in all three resistant cancer cell lines was decreased (Figure 2(a)), and the glucose consumption assay showed that the amount of glucose remaining in the culture medium was higher in HCC827 GR cells compared with HCC827 parental cells (Figure 2(b)). Using the XFp analyzer with a glycolysis stress kit, changes in the extracellular acidification rate (ECAR) representing glycolysis were estimated. After being exposed to the same amount of glucose $(10 \mathrm{mM})$, HCC 827 GR and H1993 ER cells showed less changes compared with their parental cells (Figure 2(c)). Furthermore, expression levels of enzymes involved in glycolysis ( $\mathrm{HK} 1, \mathrm{HK} 2$, and GAPDH) were lower in the two resistant cell lines (HCC827 GR and H1993 ER) (Figure 2(d)), whereas the dimer (inactive) form of Tyr105-phosphorylated PKM2 was slightly reduced in HCC827 GR and H1993 ER cells (Figure 2(d)). Notably, H292 ER cells lacked both HK1 and HK2, first-step enzymes in glycolysis, and the Tyr105-phosphorylated PKM2 level was upregulated (Figure 2(d)), indicating that glycolysis dysfunction in the H292 ER cells was mainly due to $\mathrm{HK} 1 / 2$ defects. The mRNA expression associated with the glycolysis gene set was further analyzed using RNA sequencing for both HCC827 and HCC827 GR cells. However, mRNA expression patterns associated with glycolysis in the two cell types did not exhibit any specific trends (Figure S1A). Although metabolic differences were not clearly identified at the mRNA or protein level in glycolytic enzymes, the metabolic data indicate that glycolysis capacity was decreased in the lung cancer cells with acquired EGFR-TKI resistance.

\subsection{Reactivation of Mitochondrial OXPHOS Function in} Lung Cancer Cells with Acquired EGFR-TKI Resistance. Because glycolysis is the main energy process using glucose in rapidly growing cancers, glycolysis is considered a hallmark of cancer (Warburg effect) [10]. However, the role of mitochondrial OXPHOS in cancer progression has also been studied $[4,5,11,12]$. Because EGFR inhibition induces the reactivation of mitochondrial OXPHOS in NSCLC cells [9], and the lung cancer cells with acquired EGFR-TKI resistance exhibited decreased EGFR kinase signaling activities (Figure 1(d)), OXPHOS activities in the three lung cancer cell lines with acquired EGFR-TKI resistance were compared with those in their parental cell lines. Using the XFp analyzer with a cell mito stress test kit, oxygen consumption rate (OCR) changes were monitored after treatment with OXPHOS modulators $(1.5 \mu \mathrm{M}$ oligomycin, $0.5 \mu \mathrm{M}$ trifluoromethoxy carbonylcyanide phenylhydrazone (FCCP), and a mixture of $0.5 \mu \mathrm{M}$ rotenone and $0.5 \mu \mathrm{M}$ antimycin A). ATP-linked mitochondrial respiration increased in HCC827 GR and H1993 ER cells compared with their parental cells (Figure 3(a)). In H292 ER cells, maximal respiration in mitochondria increased but ATPassociated respiration did not change significantly compared with the parental cells (Figure 3(a)). Then, the ATP production ratio was compared between glycolysis and OXPHOS using the XFp analyzer with an ATP real-time rate assay kit. ATP production in the HCC827 cells was highly dependent on glycolysis (88.75\%), but not on OXPHOS (11.25\%). However, HCC827 GR cells had more ATP production that depended on mitochondrial OXPHOS (27.14\%) than did HCC827 cells (Figure 3(b)). Mitochondrial membrane potential is induced by a proton pump in OXPHOS and regarded as an essential component during mitochondrial ATP production. Mitochondrial membrane potential in HCC827 and HCC827 GR cells was analyzed via staining with tetramethylrhodamine methyl ester (TMRM, red dots). As shown in Figure 3(c), total integrated red fluorescence intensity versus cell confluence 

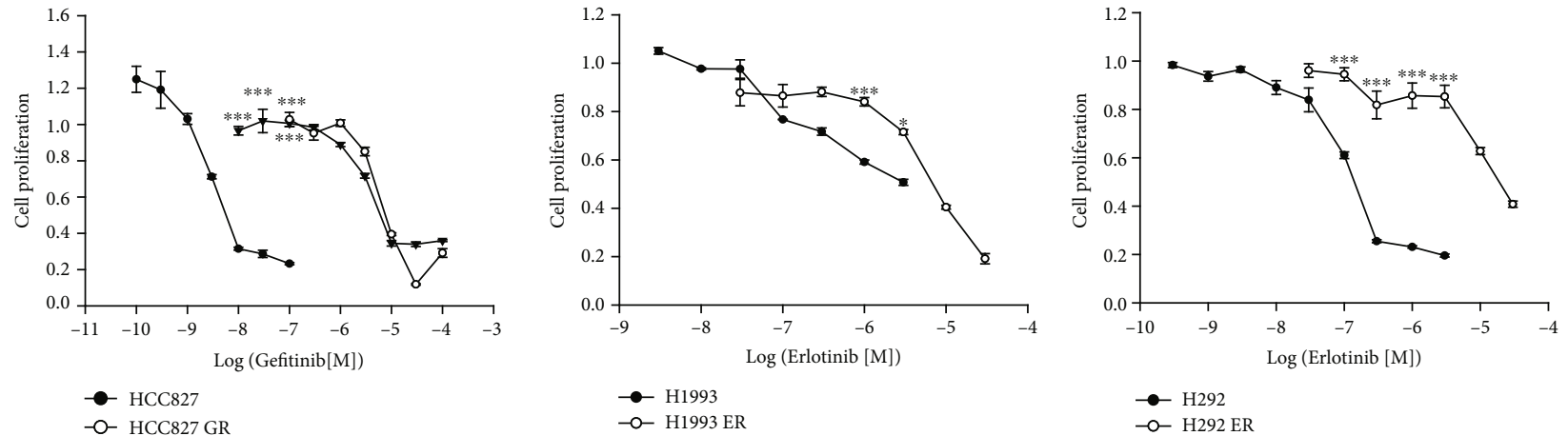

- $\mathrm{HCC} 827$

-O- HCC 827 GR

(a)
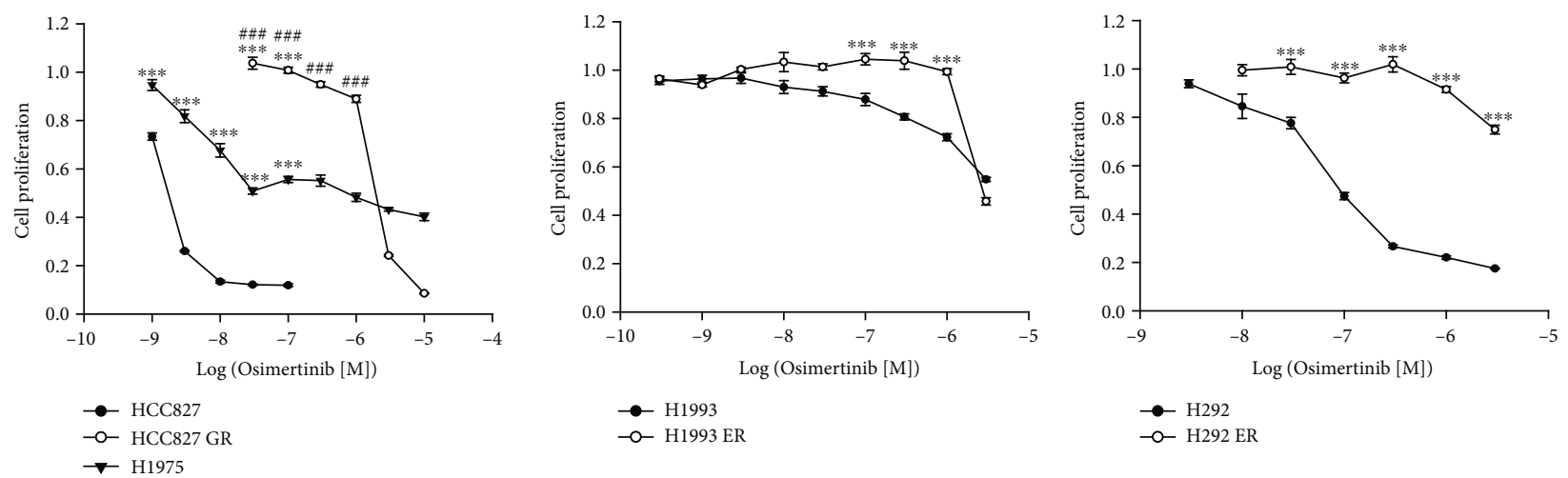

(b)

\begin{tabular}{|l|c|}
\hline & EGFR mutation (exon18 21) \\
\hline HCC827 & Del (E746-A750) \\
HCC827 GR & $\mathrm{X}$ \\
H1975 & T790M, L858R \\
& $\mathrm{X}$ \\
H1993 & $\mathrm{X}$ \\
H1993 ER & $\mathrm{X}$ \\
H292 & $\mathrm{X}$ \\
H292 ER & \\
\hline
\end{tabular}

(c)

Figure 1: Continued. 


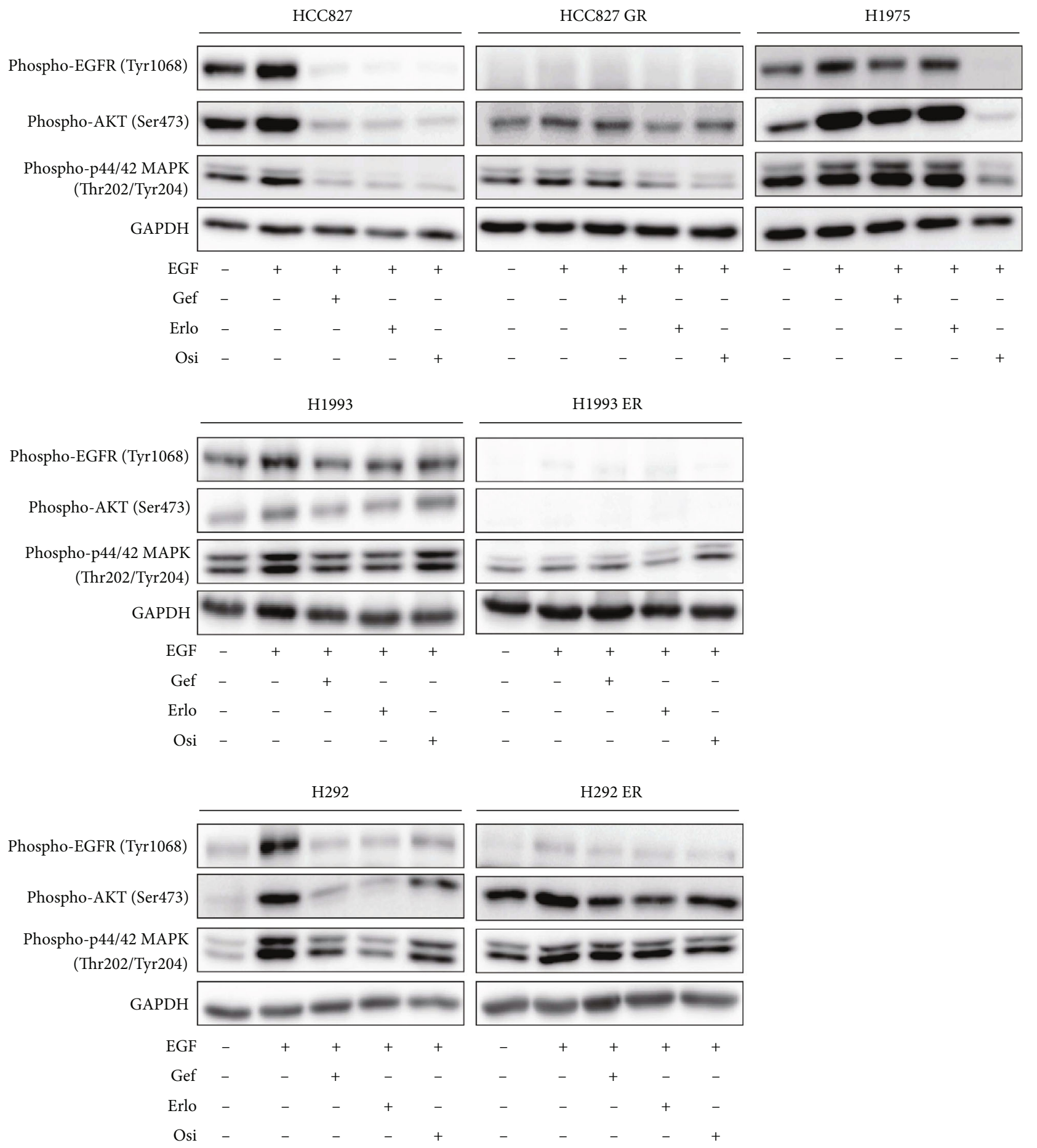

(d)

FIGURE 1: Acquisition of EGFR-TKI resistance by long-term treatment of gefitinib or erlotinib in NSCLCs. (a and b) Effects of EGFR TKIs on cell proliferation of EGFR TKI-resistant lung cancer cells. HCC827, HCC827 GR, H1993, H1993 ER, H292, H292 ER, and H1975 cells were incubated with various concentrations of gefitinib or erlotinib (a) and osimertinib (b), and cell proliferation was monitored for $72 \mathrm{~h}$ by IncuCyte ZOOM analyses. Data represent means \pm S.E.M. $(n=3-6, * P<0.05, * * P<0.01, * * * P<0.001$ vs. parental cell line; ${ }^{\# \#} P<0.001$ vs. H1975). (c) Genomic DNA sequencing of EGFR exon 18 to 21 . (d) EGFR and its downstream signaling activities in HCC827, HCC827 GR, H1993, H1993 ER, H292, and H292 ER cells. All cells were pretreated with vehicle or $100 \mathrm{nM}$ gefitinib, erlotinib, or osimertinib for $1 \mathrm{~h}$ and then exposed to $100 \mathrm{ng} / \mathrm{mL}$ EGF for $5 \mathrm{~min}$. Total cell lysates were subjected to immunoblottings for phosphoEGFR (Tyr1068), phosphor-AKT (Ser473), or phosphor-p44/p42 MAPK (Thr202/Tyr204). 

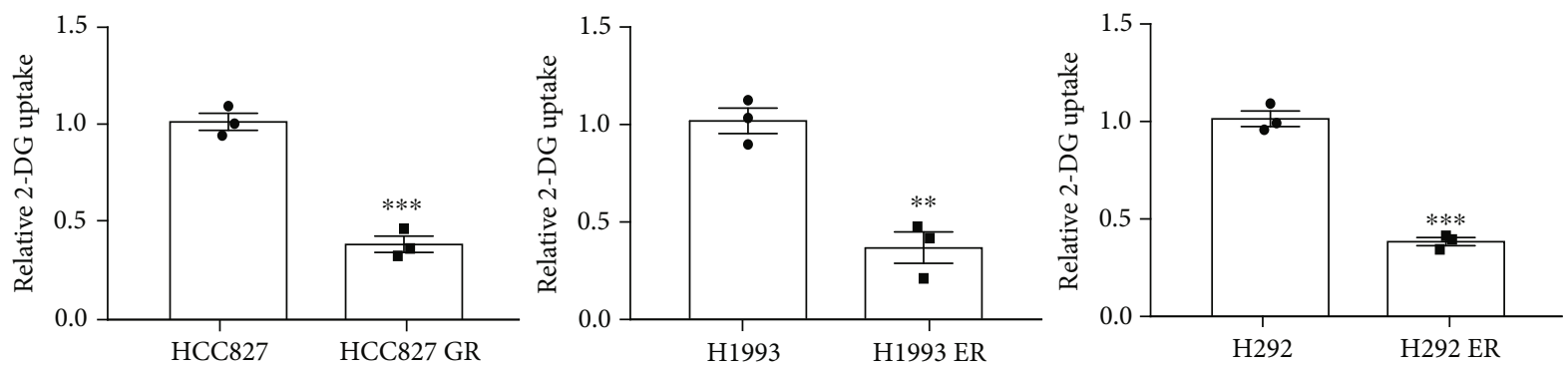

(a)

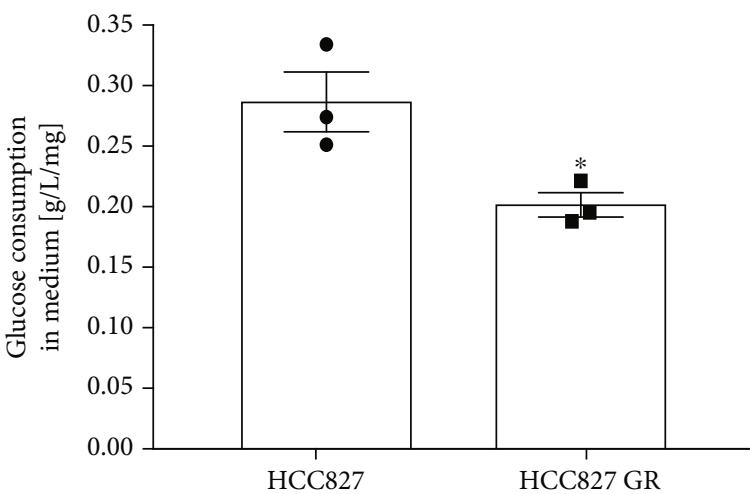

(b)
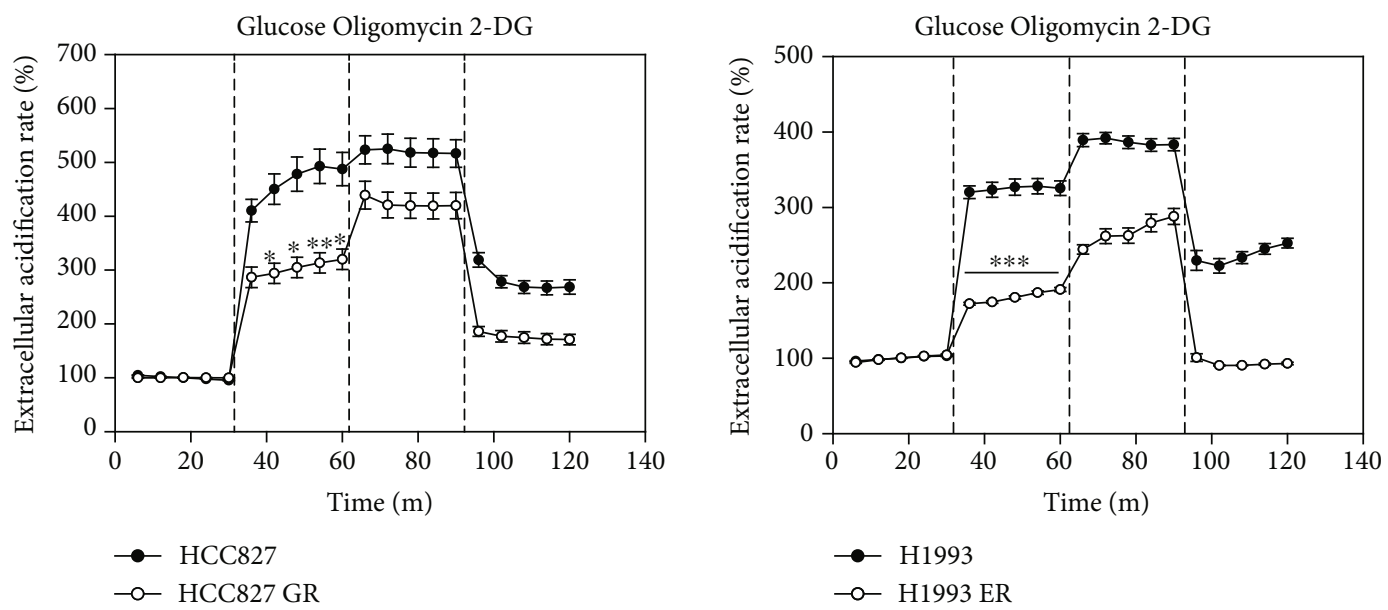

(c)

Figure 2: Continued. 

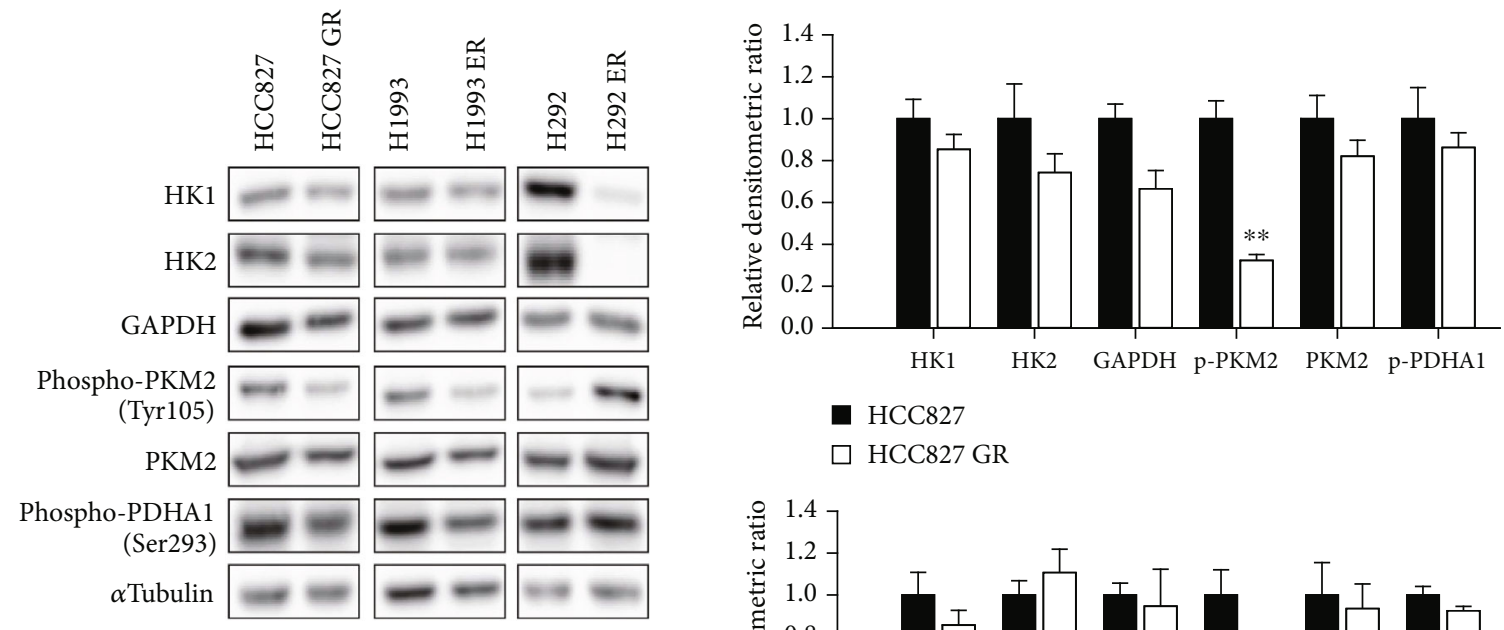

- $\mathrm{HCC} 827$

$\square$ HCC827 GR
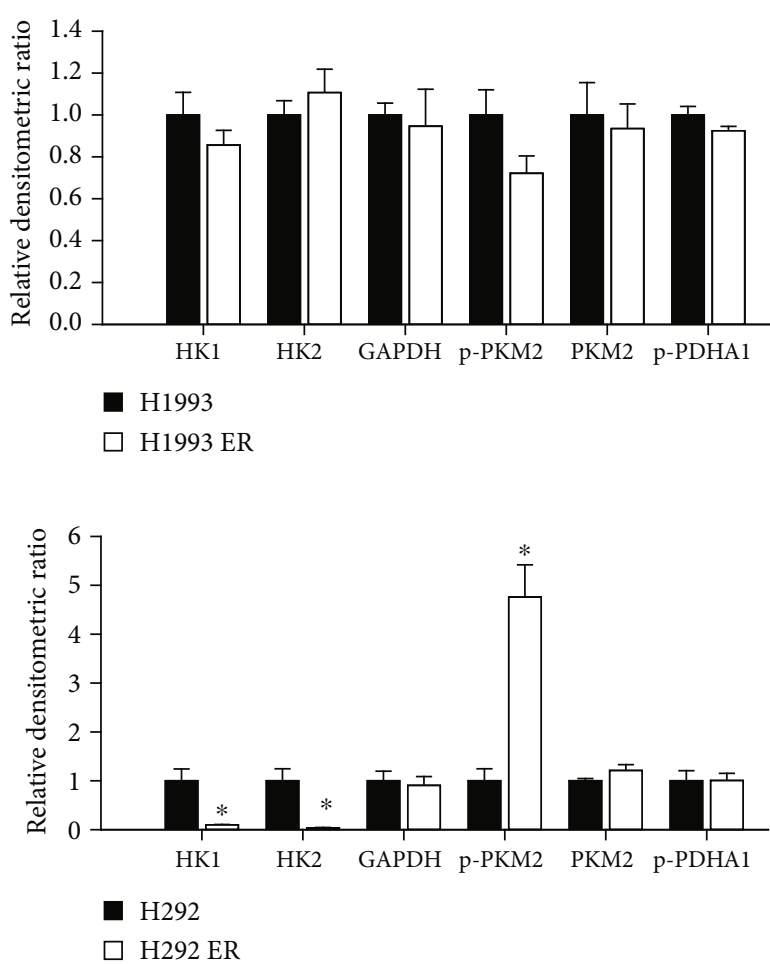

(d)

FIGURE 2: Decreased glycolysis activity in acquired EGFR-TKI-resistant lung cancer. (a) Relative glucose consumption by 2-DG uptake assay. Data represent means \pm S.E.M. $(n=3, * * P<0.01, * * * P<0.001$ vs. 2 -DG uptake in parental cell line). (b) Glucose contents in culture media. Glucose level was determined by GC-MS in culture media from HCC827 and HCC827 GR cells incubated for 24 h. Glucose concentration was normalized by total protein amounts. Data represent means \pm S.E.M. $(n=3, * P<0.05$ vs. HCC827). (c) ECAR changes. ECAR values were obtained from glycolysis stress test using XFp analyzer. Glucose (10 mM), oligomycin $(1 \mu \mathrm{M})$, and 2 -DG $(50 \mathrm{mM})$ were added at indicated time points. Data represent means \pm S.E.M. $(n=3, * P<0.05, * * P<0.01, * * * P<0.001$ vs. parental cell line). (d) Basal protein expression levels of glycolysis enzymes. Protein expression of hexokinase (HK)1, HK2, GAPDH, phosphor-PKM2 (Tyr105), PKM2, and phospho-PDHA1 (Ser293) was assessed by immunoblottings.

was significantly higher in HCC827 GR cells. Conversely, the basal intracellular level of lactate, the end product of glycolysis, was significantly decreased in HCC827 GR cells compared with HCC827 cells (Figure 3(d)). Inhibition of OXPHOS can cause a compensative increase in glycolysis resulting in the conversion of pyruvate to lactate. The lactate level increased in phenformin- (OXPHOS inhibitor-) treated HCC827 GR cells; however, a difference in HCC827 cells was not detected (Figure 3(d)). These results indicate that mitochondrial OXPHOS was reactivated in the NSCLC cell lines with acquired EGFR-TKI resistance (HCC827 GR and H1993 ER). Next, we assessed morphological changes in the mitochondria as well as the protein and mRNA expression of OXPHOS subunits. Transmission electron microscopy images showed no differences in the size and number of mitochondria in both HCC827 and HCC827 GR cells (Figure 3(e)). The expression of OXPHOS subunit proteins (complex 2, 3,5) was slightly increased in HCC827 GR cells compared with HCC827 cells; however, the OXPHOS subunits expression in H1993 ER and H292 ER cells was not significantly different from that in their parental cells (Figure 3(f)). The distribution of expression levels of different mRNAs associated with OXPHOS subunits was viewed as a MA plot. Significant fold-changes between HCC827 and 

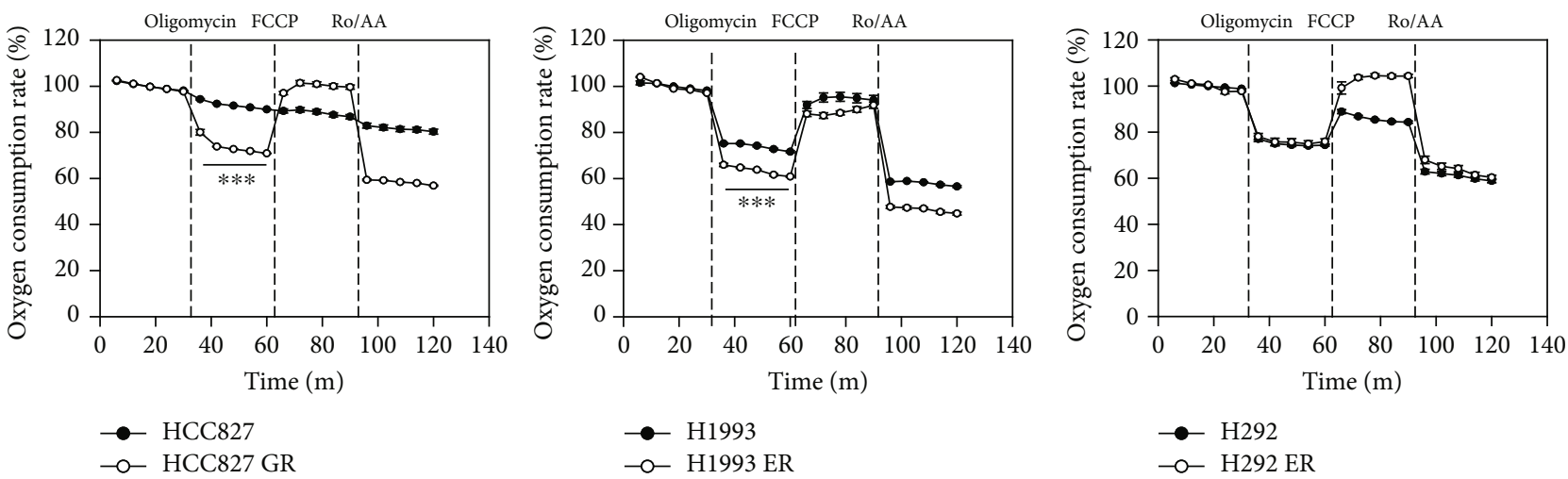

(a)

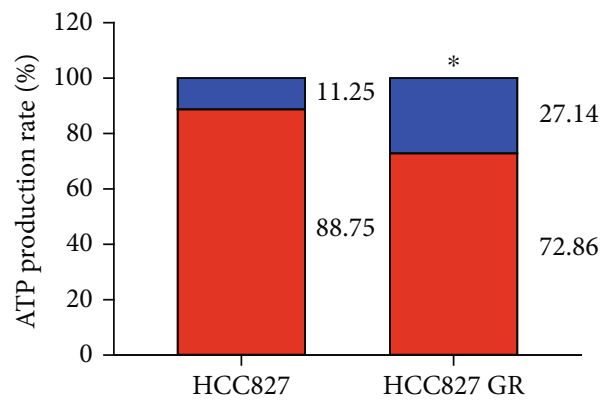

Glyco ATP production rate

Mito ATP production rate

(b)
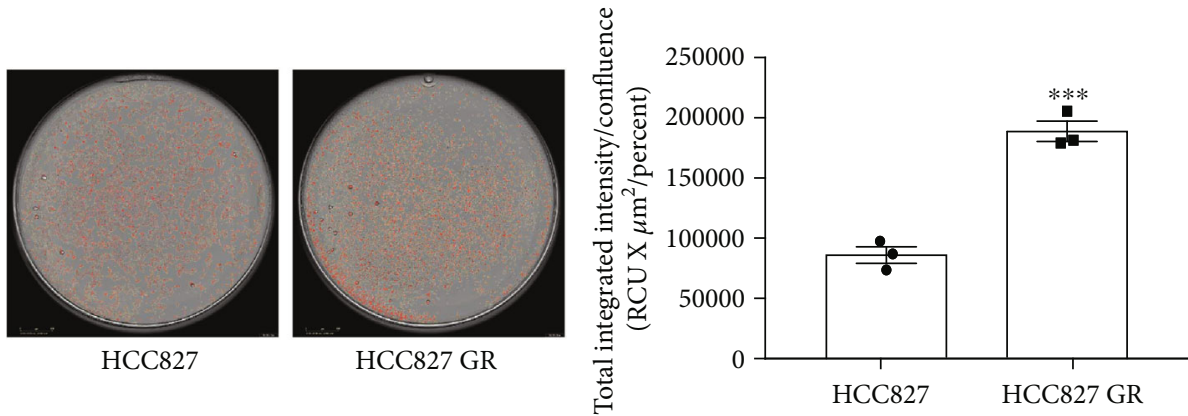

(c)
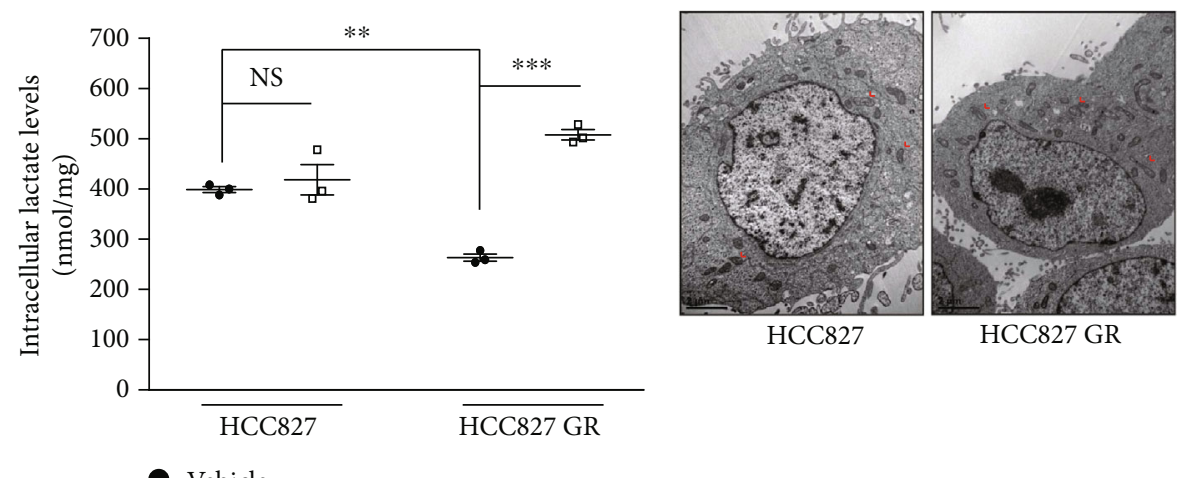

- Vehicle

$\square$ Phenformin

(d)

Figure 3: Continued. 


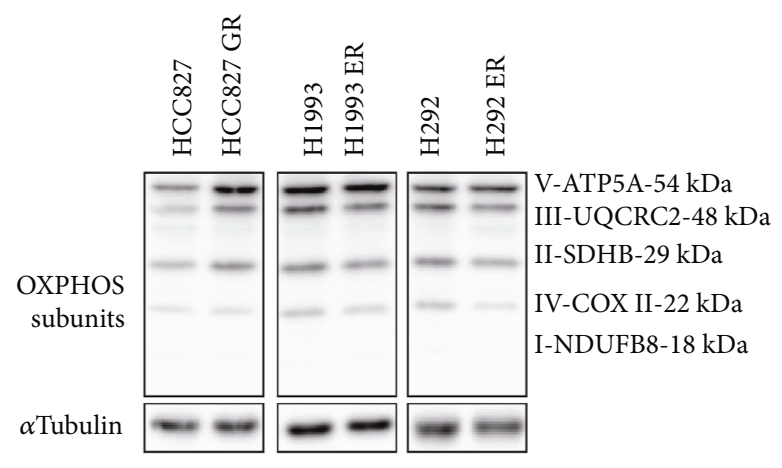

(f)

FIGURE 3: Reactivation of mitochondria function in acquired EGFR-TKI-resistant lung cancer. (a) OCR values were measured by XFp analyzer with cell mito stress kit. Oligomycin $(1.5 \mu \mathrm{M})$, FCCP $(0.5 \mu \mathrm{M})$, and mixture of rotenone (Ro, $0.5 \mu \mathrm{M})$ and antimycin A (AA, $0.5 \mu \mathrm{M})$ were treated at indicated time points. Data represent means \pm S.E.M. $(n=3, * * * P<0.001$ vs. parental cell line). (b) Relative contribution of glycolysis and OXPHOS to ATP production. Using XFp real-time ATP rate assay kit, ATP production rate from glycolysis and OXPHOS was simultaneously determined in HCC827 and HCC827 GR cells. Data represent means $(n=3, * P<0.05$ vs. mito ATP production rate in HCC827). (c) Mitochondrial membrane potential. HCC827 and HCC827 GR cells were incubated with $100 \mathrm{nM}$ TMRM for $30 \mathrm{~min}$, and fluorescence signals were detected by IncuCyte ZOOM. Total integrated intensity of TMRM (red fluorescence) was normalized with cell confluence (outlined with yellow line). Data represent means \pm S.E.M. $(n=3, * * * P<0.001$ vs. HCC827). (d) Intracellular lactate levels. HCC827 and HCC827 GR cells were treated with $100 \mu \mathrm{M}$ phenformin for $24 \mathrm{~h}$, and lactate levels in cell lysates were determined by LC-MS/MS. Intracellular lactate level was normalized with total protein amounts. Data represent means \pm S.E.M. ( $n=3, * * P<0.01, * * * P<0.001$ significant difference between the two indicated groups). (e) Number and size of mitochondria (red arrows) in HCC827 and HCC827 GR cells were analyzed by TEM. (f) Protein level of OXPHOS subunits (ATP5A, UQCRC2, SDHB, COX II, and NDUFB8) was detected by immunoblotting.

HCC827 GR cells were not found (Figure S1B). The data indicated that increased OXPHOS capacity in EGFR-TKIresistant NSCLC is associated with enhanced mitochondrial function but not with changes in related gene expression.

\subsection{Inhibition of Proliferation in Lung Cancer Cells with} Acquired EGFR-TKI Resistance Is Caused by Phenformin. Biguanides, the most prescribed anti-diabetic agents, have been recognized for their anticancer effects, and many clinical trials are currently in progress [13-16]. The repositioning of biguanides as anticancer agents is attracting much attention due to the cost benefit and minimal safety issues. In a recent study, the combination of osimertinib with phenformin delayed osimertinib resistance in a preclinical NSCLC model [17]. We hypothesized that phenformin, an OXPHOS complex 1 inhibitor, would selectively inhibit the proliferation of lung cancer cells with acquired EGFR-TKI resistance that mainly employ mitochondrial OXPHOS. A prototype biguanide, phenformin, more effectively inhibited the proliferation of HCC827 GR and H1993 ER cells than their 


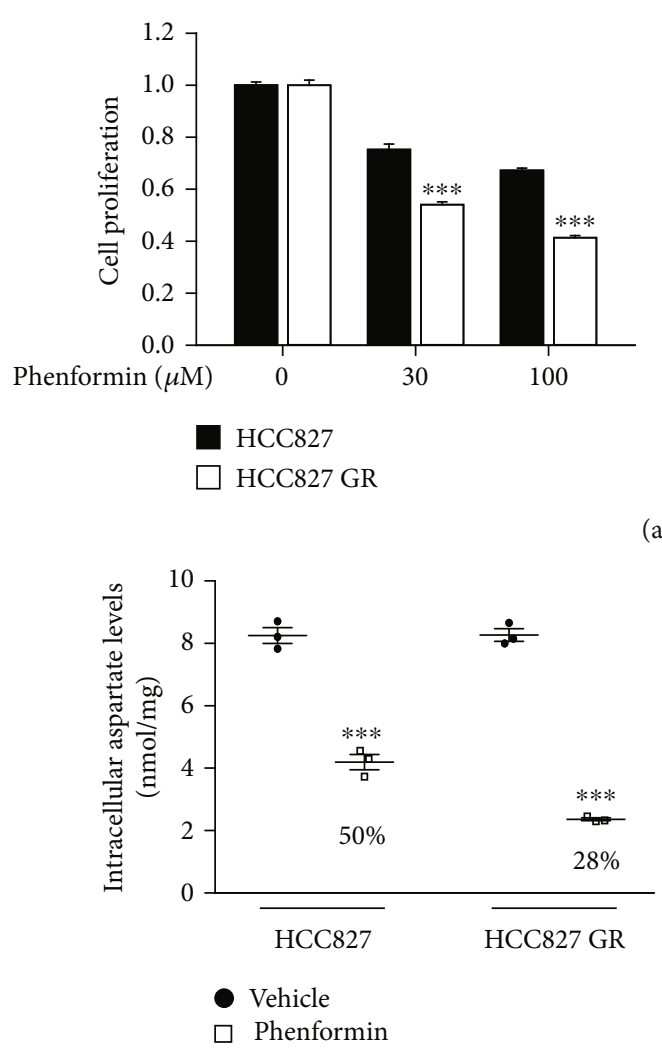

(b)

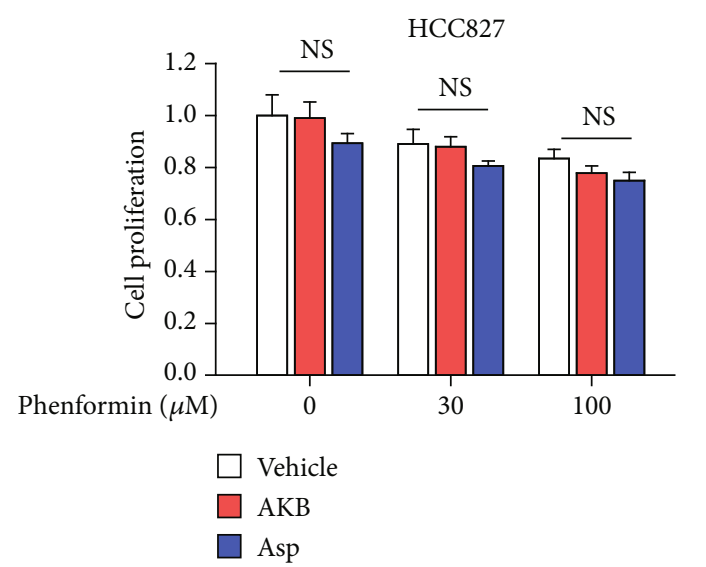

(d)

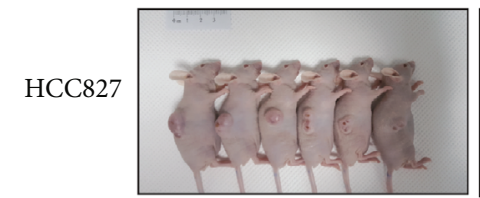

Vehicle $(n=6)$

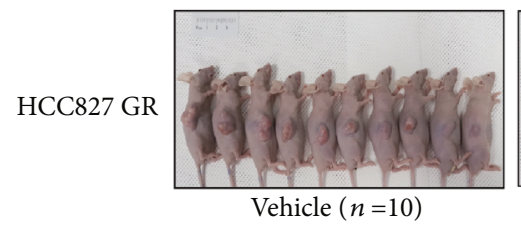

(e)

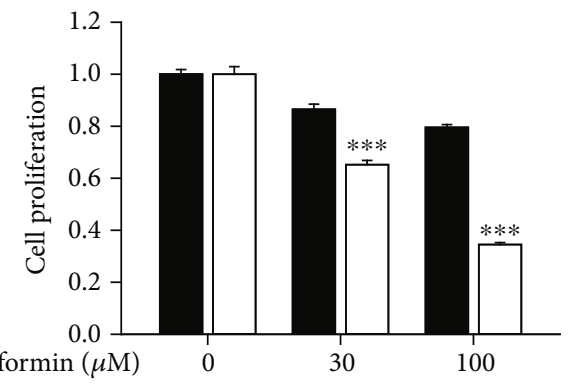

H1993

$\square$ H1993 ER

)

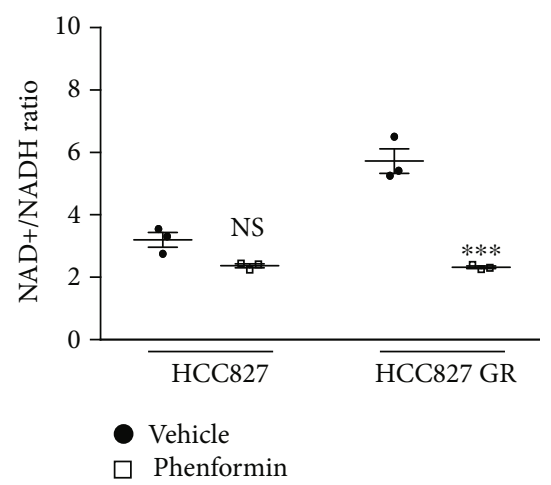

(c)

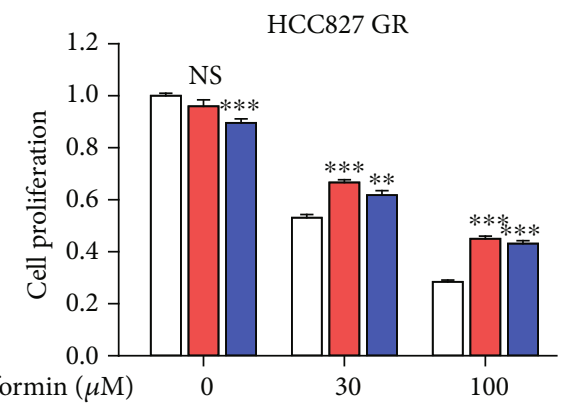

Phenformin $(n=6)$

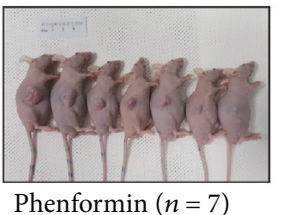

Phenformin $(n=7)$

Figure 4: Continued. 

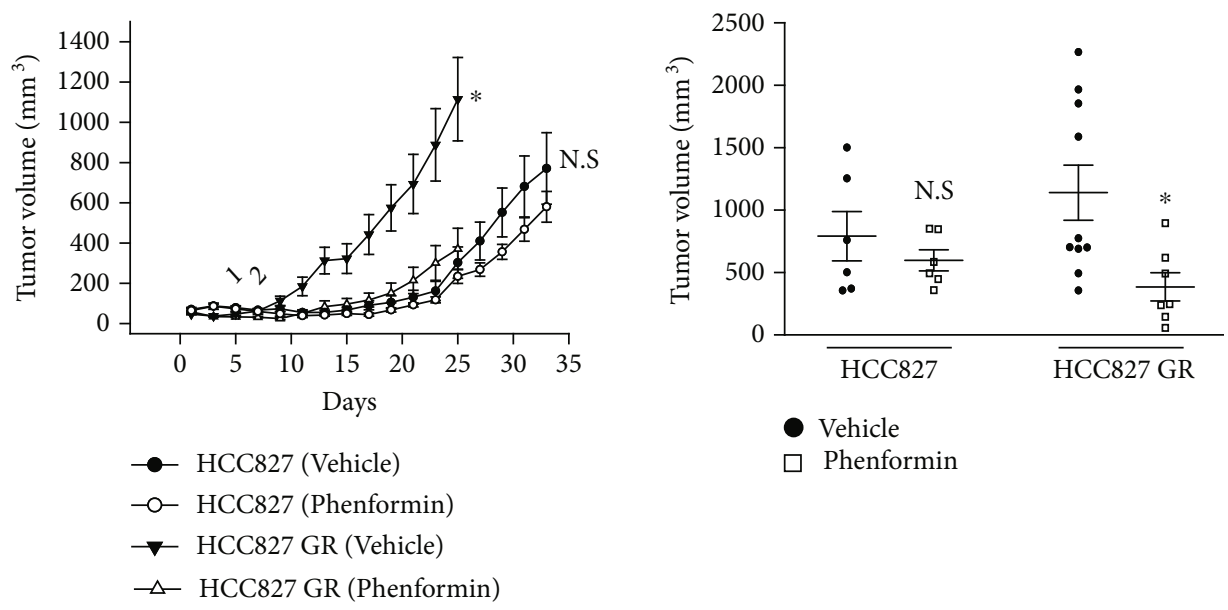

(f)

FIGURE 4: Selective anticancer effect of phenformin in acquired EGFR-TKI-resistant lung cancer. (a) HCC827, HCC827 GR, H1993, and H1993 ER cells were exposed to phenformin $(30$ and $100 \mu \mathrm{M})$ for $72 \mathrm{~h}$, and cell proliferation was monitored by IncuCyte ZOOM. Data represent means \pm S.E.M. ( $n=6, * * * P<0.001$ vs. parental cell line). (b) Intracellular aspartate level. HCC827 and HCC827 GR cells were treated with $100 \mu \mathrm{M}$ phenformin for $24 \mathrm{~h}$, and aspartate levels in cell lysates were determined by LC-MS/MS. Data represent means \pm S.E. M. ( $n=3, * * * P<0.001$ vs. vehicle-treated group). (c) Potentiation of redox stress by phenformin in acquired EGFR-TKI-resistant cancer cells. Intracellular $\mathrm{NAD}^{+} / \mathrm{NADH}$ ratio was analyzed in HCC827 and HCC827 GR cells $24 \mathrm{~h}$ after exposure with vehicle or $100 \mu \mathrm{M}$ phenformin. Data represent means \pm S.E.M. $(n=3, * * * P<0.001$ vs. vehicle-treated group). (d) Reversal of antiproliferative effect of phenformin by electron acceptor or aspartate. Cell proliferation was monitored for $72 \mathrm{~h}$ in HCC827 and HCC827 GR cells treated with phenformin $(30$ and $100 \mu \mathrm{M})$ in the presence or absence of $1 \mathrm{mM} \mathrm{AKB}$ or $10 \mathrm{mM}$ aspartate. Data represent means \pm S.E.M. $(n=6, * * P<$ $0.01, * * * P<0.001$ vs. vehicle-treated group). (e and f) In vivo anticancer effect of phenformin on tumor growth of EGFR-TKI-resistant lung cancer. HCC827 and HCC827 GR cells were inoculated into right flank of Balb/c nude mice, and the mice were orally administered with $300 \mathrm{mg} / \mathrm{kg}$ phenformin or tap water (vehicle) once a day. (e) Representative images. (f) Tumor volumes were measured every other day. Data represent means \pm S.E.M. ( $n=6-10, * P<0.05$ vs. vehicle-treated group).

parental cell lines (Figure 4(a)). However, the growthinhibiting effect of phenformin was not enhanced in $\mathrm{H} 292$ ER cells compared with H292 cells (Figure S2C), which is consistent with the data showing no difference in ATPassociated OCR change (Figure 3(a)). Next, the potential mechanism by which phenformin inhibits the proliferation of EGFR-TKI-resistant NSCLC cells was assessed. Because OXPHOS is required for aspartate biosynthesis in proliferating cells $[6,18]$, intracellular aspartate level was measured after exposing HCC827 and HCC827 GR cells to phenformin. The intracellular aspartate level decreased to a greater extent in HCC827 GR cells than in HCC827 cells after exposure to phenformin (Figure 4(b)). Inhibition of aspartate biosynthesis occurs due to an imbalance in the $\mathrm{NAD}^{+} / \mathrm{NADH}$ ratio. When the cellular $\mathrm{NAD}^{+} / \mathrm{NADH}$ ratio was measured, phenformin-mediated $\mathrm{NAD}^{+} / \mathrm{NADH}$ imbalance was only observed in HCC827 GR cells (Figure 4(c)). $\alpha$-Ketobutyrate $(\mathrm{AKB})$ is a representative electron acceptor that participates in regenerating $\mathrm{NAD}^{+}$ [6]. The addition of both $\mathrm{AKB}$ and aspartate partially alleviated the growth-inhibiting effects of phenformin in HCC827 GR cells (Figure 4(d)). To assess if mitochondrial OXPHOS complex I is a target of phenformin, the effects of rotenone, a potent OXPHOS complex 1 inhibitor, were investigated. TMRM-based mitochondria membrane potential in HCC827 GR cells was more potently reduced by rotenone treatment than HCC827 cells (Figure S2A). As expected, rotenone exerted strong growth-inhibiting effects in HCC827
GR cells, and its antiproliferative effect was reversed with $\mathrm{AKB}$ treatment (Figure S2B). To evaluate the anticancer effects of phenformin in vivo, Balb/c nude mice were implanted with HCC827 or HCC827 GR cells. In xenografts inoculated with HCC827 GR cells, oral administration of phenformin $(300 \mathrm{mg} / \mathrm{kg} /$ day $)$ significantly reduced tumor growth derived from HCC827 GR cells. Conversely, phenformin administration did not significantly affect tumor growth in xenografts inoculated with HCC827 cells (Figures 4(e) and 4(f)).

3.5. Reversal of Anticancer Effects of Phenformin Is Caused by Glycolysis Reactivation in Lung Cancer Cells with Acquired EGFR-TKI Resistance. Because the reactivation of OXPHOS in most of the lung cancer cell lines with acquired EGFRTKI resistance was observed, we hypothesized that phenformin sensitivity could be diminished by the restoration of glycolysis (Warburg effect) in the resistant cell types. Hexokinase (HK) is a first-step glycolytic enzyme that converts glucose into glucose 6-phosphate. Among the five $\mathrm{HK}$ isoforms, HK2 is highly expressed and functions as the predominant form in cancer cells [19]. Because HK2 and HK1 expressions were absent in H292 ER cells (Figure 2(d)), we hypothesized that HK enzyme deficiency is a key event for the metabolic shift to OXPHOS in H292 ER cells. HK2overexpressing H292 ER cells (H292 ER-HK2) were established by transfection with a Flag-tagged human HK2 overexpression vector (Figure 5(a)). XFp analysis with a 

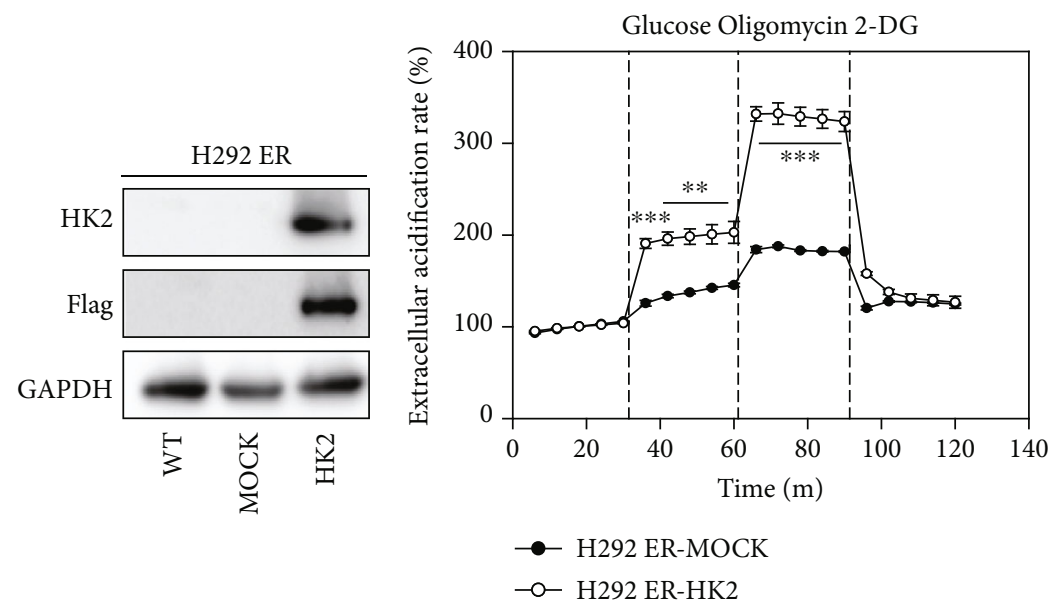

(a)

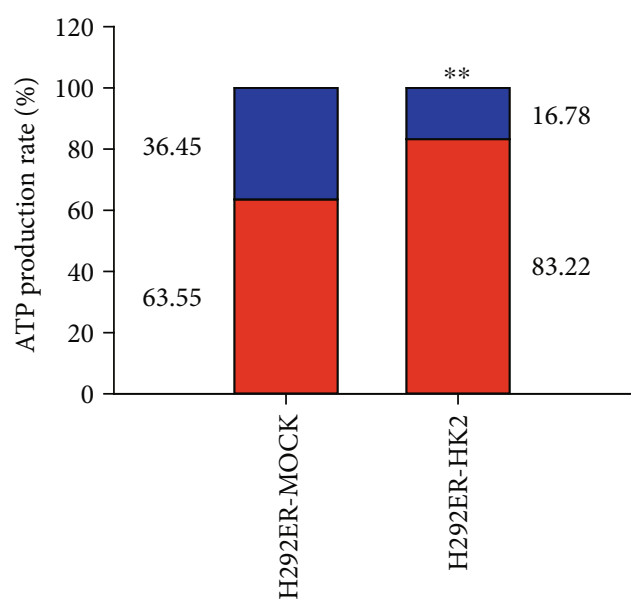

(b)

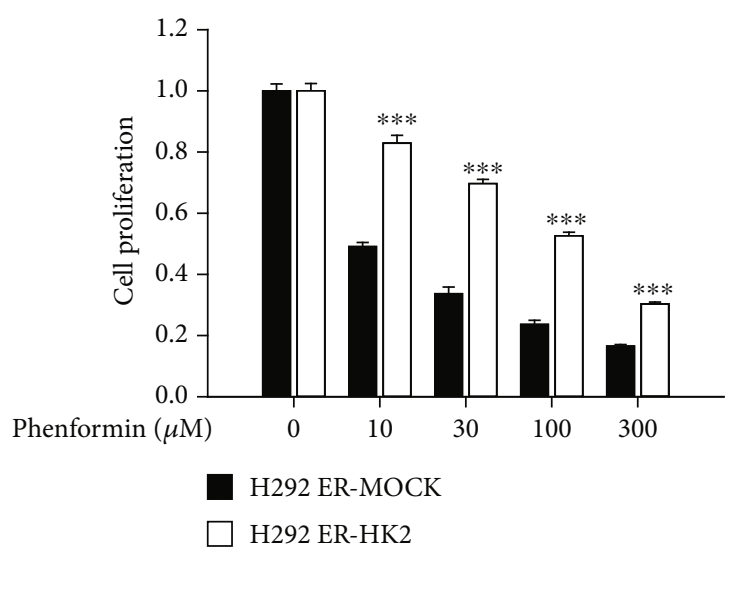

Glyco ATP production rate

$\square$ Mito ATP production rate

(c)

(d)

Figure 5: Continued. 


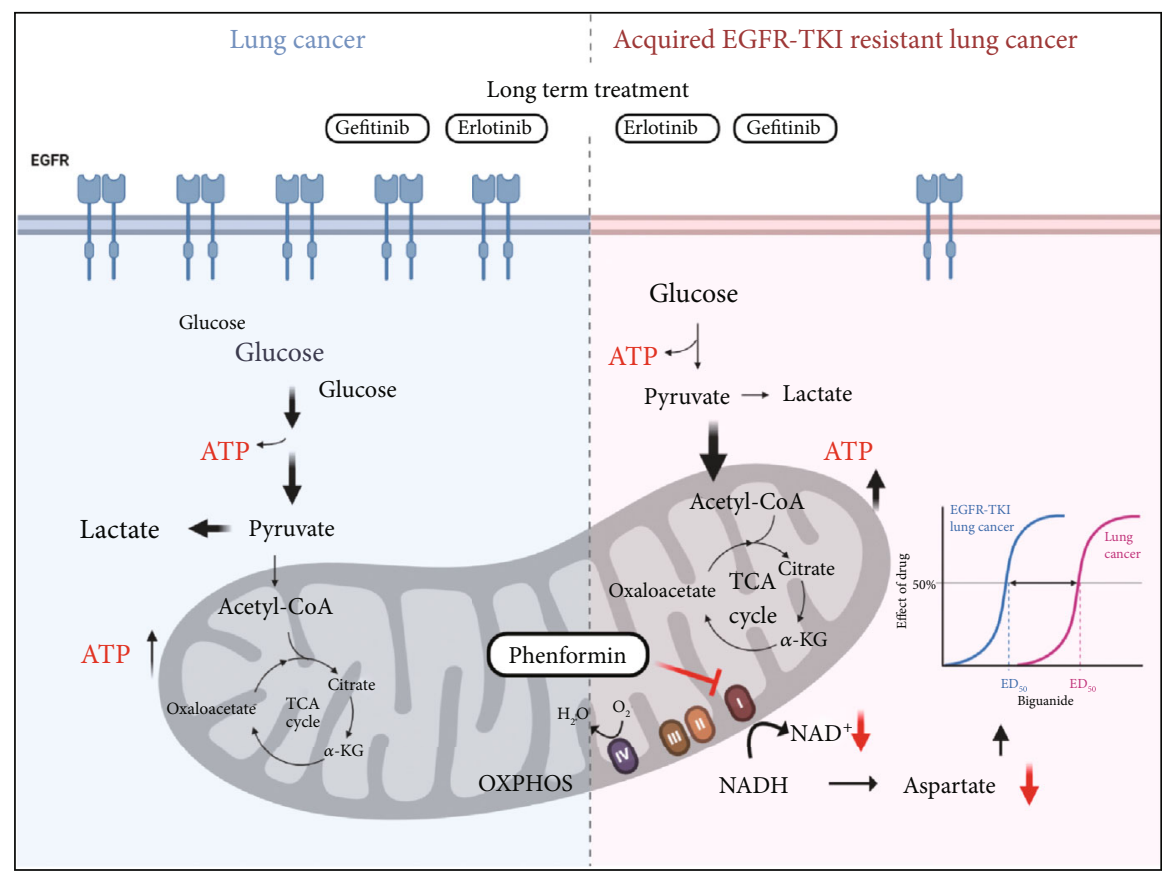

(e)

FIGURE 5: Reversal of antiproliferation effect of phenformin by glycolysis reactivation. (a) Establishment of hexokinase2 overexpressing H292 ER cells (H292 ER-HK2). H292 ER cells were transfected with pCAG-Flag-HK2-IRES-Blas or pCAG-Flag-IRES-Blas and protein expression was confirmed by immunoblotting. (b) ECAR values in H292 ER-MOCK and H292 ER-HK2 cells. Glycolysis stress test was performed by XFp analyzer, and ECAR was measured and normalized with basal ECAR level. Data represent means \pm S.E.M. $(n=3, * * P<0.01$, $* * * P<0.001$ vs. H292 ER-MOCK). (c) Relative contribution of glycolysis and OXPHOS to ATP production in H292 ER-MOCK and H292 ER-HK2 cells. Using XFp real-time ATP rate assay kit, ATP production rate from glycolysis and OXPHOS was measured in H292 ERMOCK and H292 ER-HK2 cells. Data represent means $(n=3, * * P<0.01$ vs. mito ATP production rate in H292 ER-MOCK). (d) Alleviated antiproliferative effect of phenformin by HK2 overexpression. H292 ER-MOCK and H292 ER-HK2 cells were treated with vehicle or phenformin and cell proliferation rate was monitored for $72 \mathrm{~h}$ by IncuCyte ZOOM. Data represent means \pm S.E.M. $(n=6, * * * P<0.001$ vs. H292 ER-MOCK). (e) Schematic illustration for metabolic shift to OXPHOS and the enhanced biguanide responsiveness in acquired EGFR-TKI resistant lung cancer.

glycolysis stress test kit showed that ECAR changes representing glycolysis and its capacity were greater in H292 ERHK2 cells compared with mock-transfected cells (Figure 5(b)). The ATP production ratio from glycolysis also increased from $63.55 \%$ to $83.22 \%$ (Figure 5(c)). Furthermore, the inhibitory effects of phenformin on the proliferation of H292 ER cells were significantly decreased by HK2 overexpression, indicating that enhanced glycolysis reduces the anticancer effects of phenformin in lung cancer cells with acquired EGFR-TKI resistance (Figure 5(d)).

\section{Discussion}

EGFR overexpression has been detected in approximately half of NSCLC patients and is associated with poor prognosis, whereas EGFR-TKIs such as gefitinib and erlotinib significantly increase the survival rate of patients without serious side effects (6). Unfortunately, most patients acquire resistance to EGFR-TKIs within 6-12 months [20, 21]. Several heterogeneous mechanisms, such as de novo mutation, amplification, and downstream pathway activation of EGFR or the activation of bypass pathways (e.g., pathways involving c-Met, Axl, insulin-like growth factor receptor, and other members of the EGFR family), explain the acquired EGFR-
TKI resistance in NSCLC [22-25]. Osimertinib (Tagrisso ${ }^{\circledR}$, a third-generation EGFR-TKI) has been approved for the treatment of patients with metastatic and EGFR T790M mutation-positive NSCLC [26]. However, several cases of patients resistant to osimertinib have been reported in clinics [26]. Although phenotype characteristics of EGFR-TKIresistant lung cancer cells can be classified as EGFRdependent and EGFR-independent growth [26], deciding an appropriate treatment strategy is difficult due to the acquired resistance being driven by heterogeneous mechanisms. Herein, we propose a plausible therapeutic strategy targeting the cancer metabolism of EGFR-TKI-resistant lung cancer cells that focuses on the metabolic shift to mitochondrial OXPHOS.

In the tested lung cancer cell lines, the metabolic use of glucose differed between EGFR-TKI-sensitive and EGFRTKI-resistant lung cancer cells. Both glucose uptake and glycolytic capacity were significantly reduced in lung cancer cell lines with acquired EGFR-TKI resistance compared with their parental cell lines. Conversely, the overall function of mitochondria was enhanced in the resistant cell lines, as evidenced by increases in mitochondrial ATP production and mitochondrial membrane potential. Reportedly, EGFR stimulation accelerates glycolysis in TNBC cell lines [8]. 
Furthermore, suppression of EGFR signaling in NSCLC cells reverses the Warburg effect and reactivates OXPHOS [9]. Because EGFR-TKI-resistant lung cancer cell lines (HCC827 GR, H1993 ER, and H292 ER) acquired resistance via stepwise exposure to EGFR-TKIs over a long-term period $[21,27]$ and exhibited downregulated signaling activity of EGFR (Figure 1(d)), compensatory activation of mitochondrial OXPHOS in the resistant NSCLC cell lines appears reasonable.

Cancer metabolism is an emerging research area in cancer biology. Based on the reprogrammed pathways of nutrient utilization and metabolism in cancer cells, several metabolic characteristics have been identified as hallmarks of cancer and recognized as promising therapeutic targets for cancer chemotherapy [10, 28-32]. The metabolic processes of mitochondria have attracted attention as part of the development of treatment strategies targeting cancer metabolism [5]. In particular, biguanides inhibiting mitochondrial complex I could be of interest because metformin has been used for an extended period as an effective type 2 diabetes agent, and its safety is proven [14]. However, clear evidence showing the successful anticancer efficacy of biguanides has not been reported in numerous clinical trials, which may be due to the nonselection of either a proper biomarker that determines the reactivity of biguanide or an appropriate biguanide-responding carcinoma [33-35]. In the present study, phenformin strongly inhibited cell proliferation and tumor growth in NSCLC with acquired EGFR-TKI resistance (Figure 4).

We demonstrated that the anticancer effects of phenformin depend on the metabolic status of cancer cells, ATP production via glycolysis, or mitochondrial OXPHOS (Figure 5(c)). Although diverse cellular proteins including AMP-activated protein kinase, liver kinase B1 [36], OXPHOS complex 1 [37], and mitochondrial glycerolphosphate dehydrogenase [38] have been suggested as pharmacological targets of biguanides, we considered the $\mathrm{NAD}^{+} / \mathrm{NADH}$ imbalance caused by OXPHOS complex 1 inhibition a plausible mechanism by which phenformin acts on EGFR-TKI-resistant NSCLC. Because $\mathrm{NAD}^{+}$is required to activate cytosolic malate dehydrogenase to generate oxaloacetate and then for the synthesis of aspartate through aspartate aminotransferase, a key function of mitochondrial OXPHOS and the redox balance in cancer cells is the biosynthesis of aspartic acid for the rapid growth of cancer $[6,18]$. As shown in Figure 4, the intracellular aspartate level was decreased by phenformin in EGFR-TKI-resistant NSCLC. Because the metabolic shift to mitochondrial OXPHOS is triggered by long-term exposure to EGFR-TKIs, biguanide targeting OXPHOS may result in sustained redox stress as well as a subsequent aspartic acid deficiency and can be proposed as a new therapeutic option for NSCLC with acquired EGFR-TKI resistance. Consistent with our data, the combination of phenformin with osimertinib delayed the occurrence of resistance in a preclinical model of NSCLC in a recent study [17].

The present study has several limitations. First, all lung cancer cell lines did not exhibit a metabolic shift from glycolysis to mitochondrial OXPHOS under long-term exposure to
EGFR-TKIs. In fact, phenformin sensitivity in H292 ER cells was of a similar intensity to that of its parental H292 cells (Figure S2C). Second, a proper biomarker indicating if a metabolic shift had occurred was not suggested. The expression levels of glycolytic enzymes, such as HK1, HK2, and GAPDH, were downregulated in HCC827 GR and H1993 ER cells. Conversely, Tyr105-phosphorylated PKM2, an inhibitory form of PKM2, and S293-phosphorylated PDHA1, which converts pyruvate into acetyl-CoA, were also downregulated in HCC827 GR and H1993 ER cells. Based on our results showing that the expression levels of glycolytic enzymes, such as HK1 and HK2, were low and those of the inactive form of PKM2 (Tyr105phosphorylated PKM2) and S293-phosphorylated PDHA1 were high, H292 ER cells should be more dependent on OXPHOS and more vulnerable to phenformin. However, significant differences were not observed in terms of inhibitory effects of phenformin on the proliferation of H292 and H292 ER cells (Figure S2C). Because the metabolic processes of cancer cells are diverse, elucidating an indicator that defines the metabolic characteristics of cancer is challenging. Although specific genes that uniquely control the metabolic status of cancer cells have been successfully elucidated in a few studies $[33,35,39]$, we could not find a common metabolic point for which mitochondrial function is upregulated in three different EGFR-TKI-resistant lung cancer cell lines. However, we presume that biguanide sensitivity in NSCLC with acquired EGFR-TKI resistance relies on mitochondrial OXPHOS activity. Because mitochondrial function is precisely controlled by numerous enzymes involved in several biochemical cycles, predicting the responsiveness to biguanide by only assessing specific gene expression is difficult.

\section{Conclusions}

Long-term treatment with EGFR-TKIs induces chemoresistance with a metabolic shift from glycolysis to OXPHOS in lung cancer cells. Suppressing OXPHOS by phenformin causes redox imbalance, leading to inhibition of aspartate biosynthesis and ultimately cancer cell growth. Our research provides pharmacological evidence for a therapeutic strategy using biguanides for EGFR-TKI-resistant NSCLC (Figure 5(e)).

\section{Abbreviations}

$\begin{array}{ll}\text { 2-DG: } & \text { 2-Deoxyglucose } \\ \text { AA: } & \text { Antimycin A } \\ \text { AKB: } & \text { Sodium 2-oxobutyrate } \\ \text { Asp: } & \text { Aspartate } \\ \text { ECAR: } & \text { Extracellular acidification rate } \\ \text { EGF: } & \text { Epidermal growth factor } \\ \text { EGFR: } & \text { Epidermal growth factor receptor } \\ \text { Erlo: } & \text { Erlotinib } \\ \text { FCCP: } & \text { Trifluoromethoxy carbonylcyanide } \\ & \text { phenylhydrazone } \\ \text { GAPDH: } & \text { Glyceraldehyde-3-phosphate dehydrogenase } \\ \text { Gef: } & \text { Gefitinib }\end{array}$


H1993 ER: Erlotinib-resistant H1993 cells

H292 ER: Erlotinib-resistant H292 cells

HCC827 GR: Gefitinib-resistant HCC827 cells

HK1: Hexokinase 1

HK2: Hexokinase 2

NSCLC: $\quad$ Nonsmall cell lung cancer

OCR: Oxygen consumption rate

Osi: Osimertinib

OXPHOS: Oxidative phosphorylation

PDHA1: $\quad$ Pyruvate dehydrogenase E1-alpha subunit

Phen: $\quad$ Phenformin

PKM2: $\quad$ Pyruvate kinase isozymes M2

Ro: $\quad$ Rotenone

TKI: $\quad$ Tyrosine kinase inhibitor

TMRM: $\quad$ Tetramethylrhodamine methyl ester.

\section{Data Availability}

Data is contained within the article or supplementary material.

\section{Ethical Approval}

Animal studies were approved by Seoul National University Institutional Animal Care and Use Committee (Approval \#: SNU-170717-6-1).

\section{Conflicts of Interest}

The authors declare that they have no competing interests.

\section{Authors' Contributions}

KWK and SK designed experiments and wrote the manuscript. WKK helped analyzing RNA sequencing results. SKK and SWK helped determination of cell metabolites. SK, JHI, YJC, JYL, and SJK performed experiments.

\section{Acknowledgments}

We thank Dr. Sang Kook Lee (Seoul National University, Seoul, Republic of Korea) for donating human lung cancer cells H1993, H1993 ER, H292, and H292 ER cells and Dr. Hong-Duk Youn (Department of Molecular Medicine \& Biopharmaceutical Sciences, Graduate School of Convergence Science, Seoul National University, Seoul, Republic of Korea) for donating pCAG-Flag-IRES-Blas and pCAGFlag-HK2-IRES-Blas plasmids. This manuscript has been presented as "pre-print" in "research square" according to the following link https://assets.researchsquare.com/files/rs427767/v1/05942c81-90f7-4f20-9114-62096750cbf6.pdf. This research was funded by the National Research Foundation of Korea (NRF) grant NRF-2021R1A4A1021787.

\section{Supplementary Materials}

Supplemental Figure 1: (a) MA plot showing differential RNA expression levels in HCC827 GR versus HCC827 cells. Genes related to glycolysis were highlighted in red. (b) MA plot showing differential RNA expression in HCC827 and
HCC827 GR cells. Genes related to OXPHOS subunits were highlighted in blue. Supplemental Figure 2: (a) Effect of rotenone, a representative complex 1 inhibitor, on cell proliferation of HCC827 and HCC827 GR cells. Both the cell types were exposed to $0.1 \mu \mathrm{M}$ rotenone and/or $1 \mathrm{mM} \mathrm{AKB}$ for $72 \mathrm{~h}$, and cell proliferation was assessed by IncuCyte ZOOM. Data represent means \pm S.E.M. $(n=6, * * * P<0.001$ vs. vehicle-treated group; ${ }^{\#} P<0.05,{ }^{\# \# \#} P<0.001$ vs. rotenonetreated group). (b) Effect of rotenone on mitochondria membrane potential. HCC 827 and HCC 827 GR cells were treated with rotenone for $1 \mathrm{~h}$, and TMRM fluorescence was detected by flow cytometry. Data represent means \pm S.E.M. $(n=3, *$ $* * P<0.001$ vs. vehicle-treated HCC827 cells). (c) H292 and H292 ER cells were treated with vehicle or phenformin (30 and $100 \mu \mathrm{M}$ ) for $72 \mathrm{~h}$, and cell proliferation was analyzed with IncuCyte ZOOM. Data represent means \pm S.E.M. $(n=6$ ). (Supplementary Materials)

\section{References}

[1] G. Recondo, F. Facchinetti, K. A. Olaussen, B. Besse, and L. Friboulet, "Making the first move in _EGFR_-driven or _ALK_ -driven NSCLC: first- generation or next-generation TKI?," Nature Reviews Clinical Oncology, vol. 15, no. 11, pp. 694-708, 2018.

[2] H. A. Yu, M. E. Arcila, N. Rekhtman et al., "Analysis of tumor specimens at the time of acquired resistance to EGFR-TKI therapy in 155 patients with EGFR-mutant lung cancers," Clinical Cancer Research, vol. 19, no. 8, pp. 2240-2247, 2013.

[3] "FDA resources for Information. Food and Drug Administration Web site," 2018, https://www.accessdata.fda.gov/ drugsatfda_docs/label/2018/208065s008lbl.pdf.

[4] R. J. DeBerardinis and N. S. Chandel, "Fundamentals of cancer metabolism," Science Advances, vol. 2, no. 5, article e1600200, 2016.

[5] T. M. Ashton, W. G. McKenna, L. A. Kunz-Schughart, and G. S. Higgins, "Oxidative phosphorylation as an emerging target in cancer therapy," Clinical Cancer Research, vol. 24, no. 11, pp. 2482-2490, 2018.

[6] L. B. Sullivan, D. Y. Gui, A. M. Hosios, L. N. Bush, E. Freinkman, and M. G. Vander Heiden, "Supporting aspartate biosynthesis is an essential function of respiration in proliferating cells," Cell, vol. 162, no. 3, pp. 552-563, 2015.

[7] S. V. Sharma, D. W. Bell, J. Settleman, and D. A. Haber, "Epidermal growth factor receptor mutations in lung cancer," Nature Reviews Cancer, vol. 7, no. 3, pp. 169-181, 2007.

[8] S. O. Lim, C. W. Li, W. Y. Xia et al., "EGFR signaling enhances aerobic glycolysis in triple-negative breast cancer cells to promote tumor growth and immune escape," Cancer Research, vol. 76, no. 5, pp. 1284-1296, 2016.

[9] V. De Rosa, F. Iommelli, M. Monti et al., "Reversal of Warburg effect and reactivation of oxidative phosphorylation by differential inhibition of EGFR signaling pathways in non-small cell lung cancer," Clinical Cancer Research, vol. 21, no. 22, pp. 5110-5120, 2015.

[10] D. Hanahan and R. A. Weinberg, "Hallmarks of cancer: the next generation," Cell, vol. 144, no. 5, pp. 646-674, 2011.

[11] C. T. Hensley, B. Faubert, Q. Yuan et al., "Metabolic heterogeneity in human lung tumors," Cell, vol. 164, no. 4, pp. 681-694, 2016. 
[12] C. Lehuede, F. Dupuy, R. Rabinovitch, R. G. Jones, and P. M. Siegel, "Metabolic plasticity as a determinant of tumor growth and metastasis," Cancer Research, vol. 76, no. 18, pp. 5201$5208,2016$.

[13] K. M. Schuler, B. S. Rambally, M. J. DiFurio et al., “Antiproliferative and metabolic effects of metformin in a preoperative window clinical trial for endometrial cancer," Cancer Medicine, vol. 4, no. 2, pp. 161-173, 2015.

[14] A. Vancura, P. L. Bu, M. Bhagwat, J. Zeng, and I. Vancurova, "Metformin as an anticancer agent," Trends in Pharmacological Sciences, vol. 39, no. 10, pp. 867-878, 2018.

[15] S. Niraula, R. J. O. Dowling, M. Ennis et al., "Metformin in early breast cancer: a prospective window of opportunity neoadjuvant study," Breast Cancer Research and Treatment, vol. 135, no. 3, pp. 821-830, 2012.

[16] A. M. Joshua, V. E. Zannella, M. R. Downes et al., “A pilot 'window of opportunity' neoadjuvant study of metformin in localised prostate cancer," Prostate Cancer and Prostatic Diseases, vol. 17, no. 3, pp. 252-258, 2014.

[17] M. J. Martin, C. Eberlein, M. Taylor, S. Ashton, D. Robinson, and D. Cross, "Inhibition of oxidative phosphorylation suppresses the development of osimertinib resistance in a preclinical model of EGFR-driven lung adenocarcinoma," Oncotarget, vol. 7, no. 52, pp. 86313-86325, 2016.

[18] K. Birsoy, T. Wang, W. W. Chen, E. Freinkman, M. AbuRemaileh, and D. M. Sabatini, "An essential role of the mitochondrial electron transport chain in cell proliferation is to enable aspartate synthesis," Cell, vol. 162, no. 3, pp. 540-551, 2015.

[19] D. DeWaal, V. Nogueira, A. R. Terry et al., "Hexokinase-2 depletion inhibits glycolysis and induces oxidative phosphorylation in hepatocellular carcinoma and sensitizes to metformin," Nature Communications, vol. 9, no. 1, p. 446, 2018.

[20] F. Morgillo, C. M. Della Corte, M. Fasano, and F. Ciardiello, "Mechanisms of resistance to EGFR-targeted drugs: lung cancer," Esmo Open, vol. 1, no. 3, article e000060, 2016.

[21] D. Kim, D. H. Bach, Y. H. Fan et al., "AXL degradation in combination with EGFR-TKI can delay and overcome acquired resistance in human non-small cell lung cancer cells," Cell Death \& Disease, vol. 10, no. 5, p. 361, 2019.

[22] J. A. Engelman, K. Zejnullahu, T. Mitsudomi et al., "MET amplification leads to gefitinib resistance in lung cancer by activating ERBB3 signaling," Science, vol. 316, no. 5827, pp. 1039-1043, 2007.

[23] Z. F. Zhang, J. C. Lee, L. P. Lin et al., “Activation of the AXL kinase causes resistance to EGFR-targeted therapy in lung cancer," Nature Genetics, vol. 44, no. 8, pp. 852-860, 2012.

[24] F. Morgillo, J. K. Woo, E. S. Kim, W. K. Hong, and H. Y. Lee, "Heterodimerization of insulin-like growth factor receptor/epidermal growth factor receptor and induction of survivin expression counteract the antitumor action of erlotinib," Cancer Research, vol. 66, no. 20, pp. 10100-10111, 2006.

[25] K. Takezawa, V. Pirazzoli, M. E. Arcila et al., "HER2Amplification: a potential mechanism of acquired resistance to EGFR inhibition in EGFR-mutant lung cancers that lack the Second-SiteEGFRT790MMutation," Cancer Discovery, vol. 2, no. 10 , pp. 922-933, 2012.

[26] A. Leonetti, S. Sharma, R. Minari, P. Perego, E. Giovannetti, and M. Tiseo, "Resistance mechanisms to osimertinib in _EGFR_-mutated non-small cell lung cancer," British Journal of Cancer, vol. 121, no. 9, pp. 725-737, 2019.
[27] D. H. Bach, D. Kim, S. Y. Bae et al., "Targeting Nicotinamide $N$-Methyltransferase and miR-449a in EGFR-TKI- Resistant Non-Small-Cell Lung Cancer Cells," Molecular TherapyNucleic Acids, vol. 11, pp. 455-467, 2018.

[28] B. J. Altman, Z. E. Stine, and C. V. Dang, "From Krebs to clinic: glutamine metabolism to cancer therapy," Nature Reviews Cancer, vol. 16, no. 10, pp. 619-634, 2016.

[29] R. Bajpai and M. Shanmugam, “Targeting cancer metabolism through synthetic lethality-based combinatorial treatment strategies," Current Opinion in Oncology, vol. 30, no. 5, pp. 338-344, 2018.

[30] R. J. Klement, "The emerging role of ketogenic diets in cancer treatment," Current Opinion in Clinical Nutrition and Metabolic Care, vol. 22, no. 2, pp. 129-134, 2019.

[31] A. Luengo, D. Y. Gui, and M. G. Vander Heiden, "Targeting metabolism for cancer therapy," Cell Chemical Biology, vol. 24, no. 9, pp. 1161-1180, 2017.

[32] C. M. Cheng, F. Geng, X. Cheng, and D. L. Guo, "Lipid metabolism reprogramming and its potential targets in cancer," Cancer Communications, vol. 38, no. 1, p. 27, 2018.

[33] K. Birsoy, R. Possemato, F. K. Lorbeer et al., "Metabolic determinants of cancer cell sensitivity to glucose limitation and biguanides," Nature, vol. 508, no. 7494, pp. 108-112, 2014.

[34] D. Y. Gui, L. B. Sullivan, A. Luengo et al., "Environment dictates dependence on mitochondrial complex I for NAD+ and aspartate production and determines cancer cell sensitivity to metformin," Cell Metabolism, vol. 24, no. 5, pp. 716-727, 2016.

[35] D. B. Shackelford, E. Abt, L. Gerken et al., "LKB1 inactivation dictates therapeutic response of non-small cell lung cancer to the metabolism drug phenformin," Cancer Cell, vol. 23, no. 2, pp. 143-158, 2013.

[36] R. J. Shaw, K. A. Lamia, D. Vasquez et al., "The kinase LKB1 mediates glucose homeostasis in liver and therapeutic effects of metformin," Science, vol. 310, no. 5754, pp. 1642-1646, 2005.

[37] H. R. Bridges, A. J. Y. Jones, M. N. Pollak, and J. Hirst, "Effects of metformin and other biguanides on oxidative phosphorylation in mitochondria," Biochemical Journal, vol. 462, no. 3, pp. 475-487, 2014.

[38] S. Thakur, B. Daley, K. Gaskins et al., "Metformin targets mitochondrial glycerophosphate dehydrogenase to control rate of oxidative phosphorylation and growth of thyroid cancer in vitro and in vivo," Clinical Cancer Research, vol. 24, no. 16, pp. 4030-4043, 2018.

[39] J. Chiche, J. Reverso-Meinietti, A. Mouchotte et al., "GAPDH expression predicts the response to R-CHOP, the tumor metabolic status, and the response of DLBCL patients to metabolic inhibitors," Cell Metabolism, vol. 29, no. 6, pp. 1243-1257.e10, 2019. 